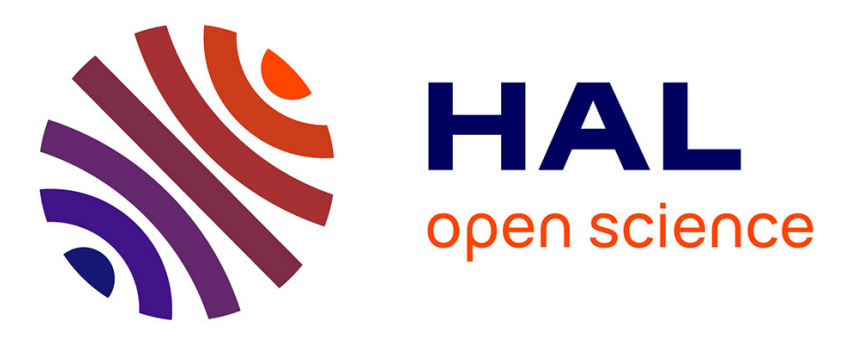

\title{
Why do farmers not convert to organic farming? Modeling conversion to organic farming as a major change
}

Qing Xu, Sylvie Huet, Christophe Poix, Isabelle Boisdon, Guillaume Deffuant

\section{- To cite this version:}

Qing Xu, Sylvie Huet, Christophe Poix, Isabelle Boisdon, Guillaume Deffuant. Why do farmers not convert to organic farming? Modeling conversion to organic farming as a major change. Natural Resource Modeling, 2018, 31 (3), pp.e12171. 10.1111/nrm.12171 . hal-02063212

HAL Id: hal-02063212

https://hal.uca.fr/hal-02063212

Submitted on 16 May 2020

HAL is a multi-disciplinary open access archive for the deposit and dissemination of scientific research documents, whether they are published or not. The documents may come from teaching and research institutions in France or abroad, or from public or private research centers.
L'archive ouverte pluridisciplinaire HAL, est destinée au dépôt et à la diffusion de documents scientifiques de niveau recherche, publiés ou non, émanant des établissements d'enseignement et de recherche français ou étrangers, des laboratoires publics ou privés. 


\title{
Why do farmers not convert to organic farming? Modeling conversion to organic farming as a major change
}

\author{
Xu Qing \\ Université Clermont Auvergne, AgroParisTech, Inra, Irstea, VetAgro Sup, UMR Territoires, \\ 63000 Clermont-Ferrand, France
}

Huet Sylvie

Lisc - Irstea, 9 avenue Blaise Pascal, 63178 Aubière, France

Poix Christophe

Université Clermont Auvergne, AgroParisTech, Inra, Irstea, VetAgro Sup, UMR Territoires, 63000 Clermont-Ferrand, France

Boisdon Isabelle

Université Clermont Auvergne, AgroParisTech, Inra, Irstea, VetAgro Sup, UMR Territoires, 63000 Clermont-Ferrand, France

Deffuant Guillaume

Lisc - Irstea, 9 avenue Blaise Pascal, 63178 Aubière, France 


\title{
Why do farmers not convert to organic farming?
}

\section{Modeling conversion to organic farming as a major change}

\begin{abstract}
.
This study aims to better understand why farmers do not convert to organic farming by studying decision trajectories in a dynamic agent-based model. In this model, an agent's decision on transitioning to organic is based on the comparison between satisfaction with its current situation and potential satisfaction with an alternative farming strategy. A farmer's satisfaction was modeled by borrowing from the Theory of Reasoned Action and computed by comparing the farmer's performance over time against the farming practices of other farmers to which he/she lends great credibility ('important others'). Analysis identified five different reasons why a farmer does not change strategy. Three are due to satisfaction or recovered satisfaction with the current situation. The conversion to organic farming is effectively a major change and cannot be envisaged if the farmer is currently satisfied. Satisfaction can be recovered when evaluation by the farmer or 'important others' finds an improvement of the current situation. A farmer's decision to not convert can also be due to negative evaluations of organic farming by 'important others', or to dissatisfaction with the current situation being too transient to prompt the effort to convert.
\end{abstract}

\section{Recommendations for resource managers.}

- A farmer's decision on whether or not to convert to organic farming is deeply influenced by his/her satisfaction with the current strategy and potential satisfaction under an alternative farming strategy.

- A farmer's satisfaction is computed by comparing the farmer's performance over time against the farming practices of other farmers to which he/she lends great credibility.

- The conversion to organic farming is effectively a major change, and a farmer will not envisage a change of strategy if he/she is satisfied with their current situation.

- A farmer's satisfaction can be recovered when evaluation by the farmer or social peers finds an improvement of the current situation.

- If a farmer's credible peers have a negative assessment of organic farming or if his/her own dissatisfaction with the current situation is too short-lived, then farmer will not convert to organic farming.

\section{Key words.}

Organic farming, decision-making, major change, theory of reasoned action, agent-based model, social influence, credibility 


\section{Introduction}

The recent dairy crisis combined with increasing consumer demand for organic food have made conversion to organic farming a socially and economically interesting move for dairy farmers (Dedieu et al. 2017; Sainte-Beuve, Bougherara \& Latruffe 2011). However, many of them still do not convert. Why not? Numerous studies point out that the dynamics of the decision depend significantly on how the agents view this decision. Michelsen (2001) and Tovey (1997) highlight that ethical and lifestyle factors shape farmers' decisions on going organic. To some scholars, organic farming is not just a set of techniques and practices but also a social movement (Fairweather et al. 2009; Darnhofer, Schneeberger \& Freyer 2005; Rigby, Young \& Burton 2001; Sutherland, Gabriel, et al. 2012; Pavie, Dockès \& Echevarria 2002), and the conversion to organic farming has recently been qualified as a major change (Sutherland, Burton, et al. 2012) or a transformational adaptation (Rickards \& Howden 2012; Dowd et al. 2014). Conversion thus implies strong changes in a farmer's identity, social network and worldview, and begins with a strong need for change (Barbier, Cerf \& Lusson 2015; Sutherland, Burton, et al. 2012). More studies are needed to better understand this type of change that engages a number of social processes as well as a farmer's own changes in terms of relations with other farmers and the farmer's environment.

Agent-based modeling (Goldstone and Janssen 2005) or individual-based modeling (Grimm 1999) appear relevant and well-geared to studying such farmer dynamics. However, a look at the reviews and model development literature show that there are practically no agent-based models for a major change (Brown et al. 2017; Janssen \& van Ittersum 2007; Martin-Clouaire 2017; Robert, Thomas \& Bergez 2016). One of the most recognized social behavioral models concerning agents in relation with others and their environment is the Theory of Reasoned Action (TRA; Fishbein 1979) and its extension, the Theory of Planned Behavior (TBA; Armitage \& Conner 2001; Beedell \& Rehman 1999; Beedell \& Rehman 2000; Fielding et al. 2008; Hansson, Ferguson \& Olofsson 2012; Lynne et al. 1995). However, these models are static. Here we propose a dynamic version of TRA to define a farmer's current or potential satisfaction regarding a behavior. These satisfactions are computed by an individual-based approach in which individuals represent farmers. We aim to investigate how the socio-psychological bases of TRA can explain the non-adoption of organic farming practices.

Our dynamic model was conceived through empirical study of dairy farmers in French mountainous areas, which offer a particularly relevant context for studying the socio-psychological dimensions of farmers' evolutions. Indeed, for these farmers, located in small villages with homogeneous farming production and practices, organic farming is high-value-added (Boisdon et al. 2013; Dockès et al. 2013). The focus on these regions allows us to consider the economic issue of conversion to organic farming through a simple heuristic lens. A farmer is supposed to approximate how beneficial the conversion is through a simple observation of the "already converted" farmers. This approximation has been shown to be relevant (Bui et al. 2016; Chantre, Cerf \& Le Bail 2015; Xavier Coquil, Dedieu \& Béguin 2017; Lamine and Bellon 2009; Alavoine-Mornas and Madelrieux 2014), and organic farming practices have also been shown economically neutral or beneficial for a majority of dairy farmers (Dedieu et al. 2017; Sainte-Beuve, Bougherara \& Latruffe 2011). To gain more practical perspective, we have also interviewed a number of farming advisors and farmers from a similar region (in the departments of Loire and Haute-Loire) in France. The Loire is one of the departments in France that counts the most organic dairy farmers, yet for a long time these farmers did not benefit from an economic diagnosis of the conversion since there was no official advisor to do it. Most of them converted to organic farming without a "rational" or "official" economic impact assessment on the conversion move. Instead of focusing solely on economic issues, farmers told their stories on how they evolved in terms of strategy and practices (Barbier, Cerf \& Lusson 2015; Boisdon et al. 2013; Bui et al. 2016; Chantre, Cerf \& Le Bail 2015; Xavier Coquil, Dedieu \& Béguin 2017; Dockès et al. 2013) .

The literature on organic farming simulation models is surprisingly poor. Most classical approaches focus on static motivations and barriers related to farm characteristics, like farm size, altitude, herd size, etc. (Gardebroek 2002; Boisdon et al. 2013; Fairweather 1999; Schneeberger, Darnhofer \& Eder 2002; Dockès et al. 2013). Louhichi, Alary \& Grimaud (2004) propose a linear mathematical model to 
study how dairy farmers change techniques in different policy contexts. Such decision-making models are very relevant for technical or financial decisions that fit the farm's main management trajectory.

Previous research has used agent-based models to understand such a process. In particular, the IMAGES agent-based model (Deffuant, Huet \& Weisbuch 2002; Deffuant, Huet \& Amblard 2005) targets the diffusion of agri-environmental measures (AEM). In this model, a farmer decides to invest in assessing the economic benefit of the conversion only after reaching a certain threshold of his/her general social interest. If a farmer has a strong negative social interest in the conversion, he/she may never assess the economic interest on his/her own farm. This conclusion arises from the actual situations observed in the interviews with farmers at that time (between 1997 and 2001). The data from the European IDARI project ${ }^{1}$ show also the same properties. In IMAGES, a farmer's general social interest is influenced by his/her discussions with peers. In the chosen dynamics, this influence is conditioned by a level of proximity in opinions (Deffuant et al. 2002). A farmer can thus keep a different opinion to the majority of his/her peers, which means a farmer can adopt an alternative farming strategy if he/she holds a strong positive opinion on the alternative, even though he/she is isolated in his/her social network and embedded in a local context that holds a negative opinion on his/her new strategy. This is not in line with data and literature. Indeed, data from the European IDARI project $^{1}$ and the theory of innovation diffusion (Rogers 1983) argue that a converted agent changes relationships in order to reinforce its choice and stay well informed on its new practice. Thus, instead of keeping the same close relation with conventional farmers as before, a new organic farmer connects to other organic farmers. Moreover, in the IMAGES model, neither the current economic situation of a farm, nor a farmer's satisfaction with his/her current practice, has an impact on the motivation to seriously assess the alternative strategy. Obviously, the process does not start with a farmer's need for change.

The principles of the IMAGES model are similar to a number of theories in social psychology, including TRA (Ajzen \& Fishbein 1975). TRA proposes a model to predict behavioral intentions with two conceptually distinct sets: attitude and subjective norm. Attitude is determined by beliefs about the possible outcomes of the behavior on various issues (economy, environment, autonomy, quality, etc.) as well as the importance given to these issues by the agent. Subjective norm is about how the agent perceives the opinions of others on the evaluated behavior, and reflects social pressure that encourages or discourages the behavior. This theory, extended by TBA (Ajzen 1991), has been shown to reliably predict the adoption of a wide range of behaviors, including environmentally responsible behaviors such as recycling, composting, energy use, water conservation, and sustainable agriculture practices (Boldero 1995; J. Beedell \& Rehman 2000; Fielding et al. 2008).

Kaufmann, Stagl \& Franks (2009) couple the IMAGES project's principles of social influence with TBA (Ajzen 1991), for which they propose a dynamic version. Their model is initialized with empirical data to measure the variables in the TBA. Each component of the theory is shown by a global variable. Only the subjective norm is made dynamic with the same social influence model as in IMAGES (Deffuant et al. 2002). Personal attitude and perceived behavioral control are static.

More recently, Deffuant, Carletti \& Huet (2012) and Huet \& Deffuant (2015) propose a complementary model of social influence in which influence does not depend on the difference between two people's beliefs but on the credibility one agent gives to another. This credibility depends on one's level of esteem for another.

Olabisi, Wang \& Ligmann-Zielinska (2015) propose an exploratory agent-based model with an abstract social network to explain why there are not more farmers going organic. In their model, at the

\footnotetext{
${ }^{1}$ IDARI (QLK5-CT-2002-02718): Integrated development of agricultural and rural institutions (IDARI) in Central and Eastern European Countries.
} 
initialization, farmer agents are randomly distributed in social space and any two agents having a small enough difference in the social space can pass information. However, the social network is static.

Like Kaufmann, Stagl \& Franks (2009), we propose a dynamic model of TRA (Ajzen \& Fishbein 1975), which we use to compute a farmer's satisfaction. In our model, an agent's decision on conversion is based on comparing his/her satisfaction with current strategy against his/her potential satisfaction with an alternative farming strategy. These satisfactions are computed with attitude and subjective norm related to the current or alternative strategy. Both attitude and subjective norm are dynamic and based on the difference in performances of practices. Consistently with TRA (Ajzen \& Fishbein 1975), our model integrates decomposed variables - in this case attitude and subjective norm-instead of global variables as in Kaufmann, Stagl \& Franks (2009). Our work thus advances that a farmer's evaluation is based on his/her concrete strategy and practice instead of an abstract general opinion. The evaluation relates to his/her own experiences and his/her peers' strategies and practices.

A farmer's attitude regarding his/her current strategy is the difference between his/her performances with current strategy in memory and his/her current performance. Mintzberg, Raisinghani \& Theoret (1976) and Öhlmér, Olson \& Brehmer (1998) argue that a farmer's earlier practices are a hugely influential factor in his/her decision on a future practice.

Regarding an alternative strategy, since a farmer may have no memory about it, the attitude is the difference between his/her performances with current strategy in memory and the performances of other farmers who have already adopted the strategy or the performances of this strategy in the media.

Regarding the subjective norm, our model introduces a very different social influence process that is based on the credibility that an agent lends to another. This credibility is computed by a personal evaluation of another agent through the difference between their performances. This difference is appreciated through a value system that tells how good or bad the difference is. For example, for a conventional farmer, another farmer who produces more milk than he/she does will be evaluated positively and given high credibility. Our social influence model is closely related to the definition of professional identity and appears appropriate for the conversion to organic farming, as the conversion to organic farming is a major change that leads to deep shift in professional identity and possibly also personal values and social relations (Sutherland, Burton, et al. 2012). Note that the term "practice" in the model is not really a farmer's actual practice but the associated outcome on which it is evaluated.

Darré (1985) studied chat among dairy farmers and found that farmers co-construct their practices. Our model exemplifies the recent trend in cognitive sciences by considering individual-level cognition and behavior not in isolation but in a context of many interacting individuals (Mason, Conrey \& Smith 2007). In the model, a farmer's practice is updated by comparing it against other farmers' practices.

Another important feature of our model is tied to the decision process integrating the concept of "major change". Major change is only considered in certain situations that are critical, as the various costs involved (economic, cognitive, emotional, etc.) are so expensive. Otherwise, actors do not even consider changing their major options. In a stable period, if a farmer is satisfied enough with his/her current farming strategy, he/she does not envisage an alternative one. Only a certain level of frustration or critical event (succession, major change in the farm, etc.) will prompt a farmer consider an alternative to his/her current strategy.

Our dynamic model of TRA emerges a number of reasons why farmers do not change. In most cases, a farmer does not change because he/she is satisfied with his/her current strategy. This is often seen with farmers on large or medium farms in terms of production level. In some cases, a farmer does not change because he/she does not consider conversion as a better strategy than his/her own. In other cases, the farmer may not stay dissatisfied for long enough to decide a change strategy. Sometimes a farmer who is dissatisfied with his/her strategy can return to being satisfied due to an improvement of attitude or subjective norm. All these reasons are extracted from our dynamic model and studied in this paper. 
After presenting the principles of our model, we outline the model's behaviors and give explanations related to them, especially the reasons for not changing farming strategy, before going on to synthesizing and discuss our conclusions.

\section{Materials and Methods}

Here we describe the model: its basic elements and its dynamics.

\section{The basic elements}

\section{The farmer}

The model studies the evolution of a population composed of $N$ farmer agents. Each farmer agent is characterized by its farm, its farming strategy, its practice defined on several dimensions $i$ which are evaluated through performances (where "practice" is not really a farmer's actual practice but the associated outcome on which it is evaluated), the importance $W_{i}$ it gives to each dimension of practice, the credibility $(C(f, v))$ it lends to each other farmer, and its memory of applied strategies and practices during the last $M$ periods. Each agent can also have satisfactions with current farming strategy $\left(I_{C}\right)$ and with alternative $\left(I_{A}\right)$ farming strategy.

$D D$ and $D C$ are an agent's attributes that capture the duration between two events related to the decision process. $D D$ counts the duration of an agent's dissatisfaction with its current strategy. In the model, an agent has to be dissatisfied long enough with its current strategy to change it. $D C$ counts the duration of an agent's wait after changing strategy. After changing strategy, there is a confirmation period during which an agent cannot consider changing strategy again even if it is dissatisfied with the new one, which is consistent with innovation diffusion theory (Rogers 1983) in which an agent has a confirmation period just after adopting a new strategy. Both counters are necessary to simulate an agent's stability and consistency. The corresponding delayed action of both counters can only occur when the counter is above the parameter $T D$.

Except for $W_{i}$, all these attributes are dynamic during the simulation, and are described in detail below.

\section{Credibility}

Each agent $f$ gives a credibility $C(f, v)$ to another agent $v$ by comparing their practices. If the credibility given is below 0.01 , we consider it as null, i.e. 0 . Credibility is assumed to be between 0 (no credibility at all) and 1 (huge credibility).

\section{Satisfaction}

Each agent has a satisfaction with its current farming strategy $\left(I_{C}\right)$ that corresponds to an evaluation of that strategy. If it is dissatisfied with its current farming strategy, the agent will also evaluate its satisfaction with the alternative one $\left(I_{A}\right)$. Satisfaction with a farming strategy lies between 0 (not satisfied at all) and 1 (very satisfied).

If an agent is satisfied with its current farming strategy, then it does not consider an alternative. Otherwise, its satisfactions with current farming strategy $\left(I_{C}\right)$ and with alternative farming strategy $\left(I_{A}\right)$ are computed and compared. If $I_{A}$ is sufficiently better than $I_{C}$, the agent will change farming strategy. $I_{C}$ is thus computed at every iteration, whereas $I_{A}$ is only computed when the agent is dissatisfied with its current farming strategy.

In accordance with TRA, the satisfaction $I_{S}$ with a farming strategy $S$ depends on two elements: attitude $A_{S}$ and subjective norm $S N_{S}$ toward $S$. In the original theory, the interaction between these two elements varies with different agents facing different situations. Here, in order to keep the model simple, satisfaction is assumed to be the average value of these two elements.

$$
I_{S}=\frac{A_{S}+S N_{S}}{2}
$$


Both attitude and subjective norm lie between -1 (very negative attitude/subjective norm concerning the farming strategy to evaluate) and 1 (very positive attitude/ subjective norm concerning the farming strategy to evaluate). They are computed with the farmers' practices, strategies, and credibilities. See the section below headed "Farmers" dynamics" for details of the computations.

Considering the value range of attitude and subjective norm towards a farming strategy, satisfaction should also have been between -1 and 1 . However, to facilitate other calculations, satisfaction is normalized to between 0 and 1 .

\section{Practice}

As stated above, the term "practice" in the model is not really a farmer's actual practice, but the way he/she evaluates it. A practice is evaluated over two dimensions: level of production of the farm output (i.e. the productivity impact, in our case milk production), and the level of production of environmental amenities (i.e. the environmental impact), respectively called productivity performance $\left(P_{0}\right)$ and environmental performance $\left(P_{l}\right)$ in what follows. We assume $P_{0}$ and $P_{l}$ lie between 0 (very bad in this dimension of practice) and 1 (very good in this dimension of practice).

Importance given to each dimension of practice

The importance given to productivity performance is termed $W_{0}$, and and the importance given to environmental performance is termed $W_{l} . W_{0}$ and $W_{l}$ lie between 0 (not important at all) and 1 (most important) and they sum to one.

$$
\mathrm{W}_{0}+\mathrm{W}_{1}=1
$$

Importance here defines an agent's personal values. An agent uses its own lens to judge the information it receives and the other agents it meets. In this model, both $W_{0}$ and $W_{l}$ are kept constant if an agent does not change its farming strategy.

\section{Farming strategy}

Farming strategy is defined by initialized environmental performance $\left(P_{l}\right)$ and the farmer's importance given to each dimension of practice. Here we are dealing with two farming strategies: organic and conventional. Farmers running an organic farming strategy lend more importance to environmental performance and less importance to productivity performance, whereas farmers employing a conventional farming strategy lend more importance to productivity performance and less importance to environmental performance.

\section{Farm}

In the model, each farmer has a farm, which is very simply defined by two variables: level of production, and reference.

- Level of production is a synthetic indicator of a farm's characteristics. It defines the capacity to produce on the farm in terms of maximum level of milk production.

- Reference represents the capacity to produce on a farm under the corresponding farming strategy, and is less than or equal to level of production.

\section{Level of production}

Our model is used to study French dairy farms in mountainous areas, where practice is productioncapped by a quota system that in France has existed from 1970 (1984 in Europe) to 2015. Even since the end of the milk quotas system, a farmer's production is still more or less limited by the contract he/she has with the dairy enterprise that collects his/her milk. Under this contract, the dairy enterprise committed to buy a certain level of milk produced (that we will continue to call level of production for the sake of simplicity) at a certain price. If production is higher, the farmer gets a heavy fine in the first period (until 2015) or has to sell his/her surplus milk at a far lower price in the second period. Thus, in the model, a farm is constrained to produce less than a certain level of milk production. This maximum production is called $Q$. For the sake of simplicity, we call farms with a low level of 
production $(Q 0)$ 'small farms', with a medium level of production $(Q 1)$ 'medium farms', and with a high level of production $(Q 2)$ 'large farms'.

In this model, there is no "dairy enterprise" object considered, and level of production remains constant over the time.

\section{Reference}

On each farm, there is a reference corresponding to a farmer's capacity to produce. This reference $(R)$ depends on the farm's characteristics and farming strategy.

Analysis of the interviews and the experts' arguments shows that the reference for farms applying a conventional strategy is very similar to their level of production, whereas references for organic farms are slightly lower than their level of production. This difference between conventional and organic farms is given by the parameter $Y L D$.

Conventional farms are given the level of production $(Q)$ as reference while organic farms have a smaller reference which is considered to be $(Q(1-Y L D))$, where $Y L D$ is a parameter in the model $(0<Y L D<0.3)$.

$$
R^{f}=\left\{\begin{array}{c}
Q^{f} \text { if } f \text { applies conventional strategy } \\
\text { else } Q^{f}(1-Y L D)
\end{array}\right.
$$

\section{Media}

When an agent is dissatisfied with its current farming strategy and looks for an alternative in the population, it first searches for other agents having its same level of production but applying an alternative farming strategy. If it cannot find one in the population, it has access to the media to benefit from the idealized description of the alternative farming strategy. In the media, there is one model for each farming strategy and each level of production. Each model has the best practice (highest value for both dimensions of a practice) it can have with its farming strategy and level of production. Media is kept constant during the simulation.

The next section presents the dynamics of the model.

\section{Farmers' dynamics}

\section{Overview of a farmer's dynamics over years}

One time-step (iteration) $t->t+1$ represents one year in real life, i.e. farmers decide their farming strategies, their practices, and so on, once a year.

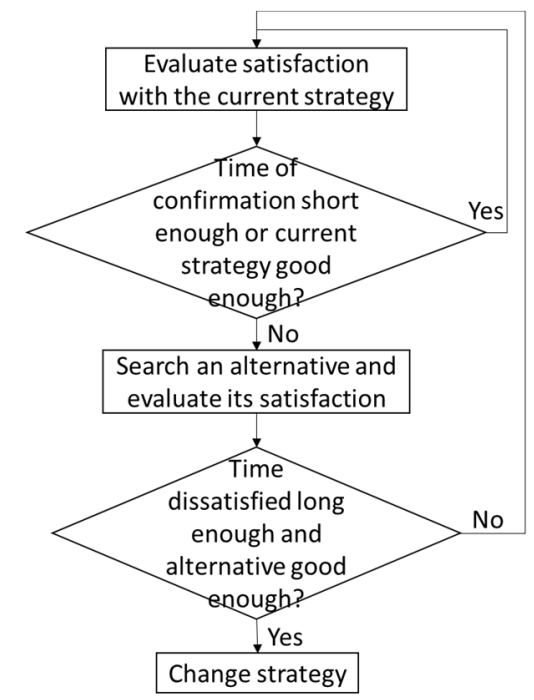

Figure 1-Overview of the farmer's update 
As shown in Figure 1 and the pseudocode of algorithm 1, during each iteration, an agent evaluates its satisfaction with current farming strategy. If the agent is in a stable period and is satisfied with the current strategy, it does not consider a change. Otherwise, the agent looks for an alternative and evaluates it. If the agent has been dissatisfied for long enough and the alternative is good enough, the agent will change. Otherwise, the agent stays with its current farming strategy. It will then update the credibility given to other agents and practices.

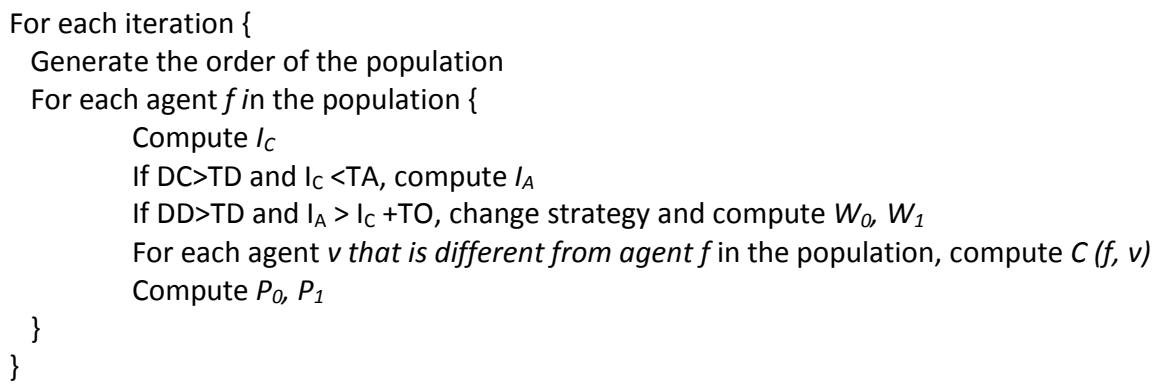

Algorithm 1- Population updating loop. $I_{C}$ is satisfaction with the current strategy, $I_{A}$ is satisfaction with the alternative strategy, $D C$ is duration of an agent's confirmation state, $D D$ is duration of an agent's dissatisfaction state, $T D$ is minimum wait-time required comparing with $D C$ and $D D, T A$ is threshold of $I_{C}$ to consider an alternative, $T O$ is threshold above $\left(I_{A}-I_{C}\right)$ to change strategy, $W_{0}$ is importance given to productivity performance, $W_{I}$ is importance given to environmental performance, $C(f, v)$ is the credibility that agent $f$ gives to agent $v, P_{0}$ is productivity performance, $P_{l}$ is environmental performance.

During an iteration, the order of farmers to be updated is picked up at random using a uniform law.

\section{Farming strategy change}

An agent changes its farming strategy according to its evaluations of satisfaction with the current farming strategy $\left(I_{C}\right)$ and with the alternative farming strategy $\left(I_{A}\right)$. As shown before, satisfaction $I$ with a strategy ( $C$ for current strategy, $A$ for alternative strategy) is the average sum of the related attitude and subjective norm. If an agent is stable, that is to say its duration of confirmation $(D C)$ is above a certain threshold $(T D)$ and it is still dissatisfied with its current farming strategy $\left(I_{C}\right.$ is less than a certain threshold $T A$ ), it will consider an alternative. If the agent's duration of dissatisfaction since being stable $(D D)$ is above $T D$ and $I_{A}$ is better than the sum of $I_{C}$ and a certain threshold $T O$, it will change farming strategy. The following shows how a farmer's attitude and subjective norm towards each strategy are computed.

In equation (1), attitude $\left(A_{s}\right)$ represents an agent's personal view of the difference between its experience and the practice to be evaluated. The agent's experience is its average practice $\left(\overline{P_{C, 0}}, \overline{P_{C, 1}}\right)$ with current farming strategy $(S)$ in its memory $(M)$. It is computed like this:

$$
\overline{P_{C, l}}=\frac{\sum_{\text {tand } S^{t}=S}^{M} P_{i}^{t}}{N b\left(S^{t}=S\right)}
$$

The practice to be evaluated depends on the strategy to be evaluated. For the evaluation of agent $f$ 's current farming strategy, the practice to be evaluated is each dimension of $f$ 's current performance $\left(P_{i}^{f}\right)$.

So, agent $f$ 's attitude toward the current farming strategy $\left(A_{C}\right)$ is like this:

$$
A_{C}^{f}=\sum_{i=1}^{2}\left(W_{i}^{f}\left(P_{i}^{f}-\overline{P_{C, l}^{f}}\right)\right)
$$

If an agent changes its practice, and this change is considered to be better than experience, $A_{C}$ will be positive and strengthen the agent's decision to keep its current strategy. Otherwise, if its practice is worse than its experience, $A_{C}$ will be negative and may influence $I_{C}$ in such a way the agent will be dissatisfied and evaluate $I_{A}$. 
For agent $f$ 's evaluation of the alternative farming strategy, the practice to be evaluated is the average practice $\left(\overline{P_{A, 0}^{f}}, \overline{P_{A, 1}^{f}}\right)$ of other agents having the same level of production as $f$ but applying the alternative farming strategy. The practice to be evaluated is computed as follows:

$$
\overline{P_{A, l}^{f}}=\frac{\sum_{Q^{v}=Q^{f} \text { and } S^{v} \neq S^{f}}^{N} P_{i}^{v}}{N b\left(Q^{v}=Q^{f} \text { and } S^{v} \neq S^{f}\right)}
$$

If there is no corresponding peer (no other agents having the same level of production as $f$ and applying the alternative farming strategy), then the agent will search the media for a stereotypical farm having a similar level of production in order to evaluate the alternative.

$$
\overline{P_{A, l}^{f}}=P_{\text {model }, i}^{f}
$$

So, agent $f$ s attitude toward an alternative is like this:

$$
A_{A}^{f}=\left\{\begin{array}{c}
0 \text { if }\left(I_{C}^{f}>T A\right) \\
\sum_{i=1}^{2}\left(W_{i}^{f}\left(\overline{P_{A, l}^{f}}-\overline{P_{C, l}^{f}}\right)\right) \text { otherwise }
\end{array}\right.
$$

Attitude is one component of satisfaction. The following shows how subjective norm, the second component, is valued.

Subjective norm represents how an agent considers others' opinions on the evaluated farming strategy. The strategy is evaluated through the agents' practices, i.e. the way they implement their strategy. Subjective norm is thus an agent's perceived difference between the practice to be evaluated and the average of other agents' practices.

For agent f's evaluation of current farming strategy, the subjective norm is:

$$
S N_{C}^{f}=\sum_{i=1}^{2}\left(W_{i}^{f}\left(P_{i}^{f}-\frac{\sum_{v \neq f}^{N}\left(C_{v}^{f} P_{i}^{v}\right)}{\sum_{v \neq f}^{N} C_{v}^{f}}\right)\right)
$$

An agent will be very socially satisfied if it perceives that other agents, especially those to whom it lends great credibility ("important others"), consider him as a 'good farmer'. An agent may be so happy to have a good image in the peer group that it will never consider changing farming strategy. Otherwise, if the agent feels in a socially bad situation, it may try to change to become like others in the peer group or to change groups to get a better image in the lens of others. So, the situation can be bad enough to provoke an agent to decide to change strategy.

For the evaluation of alternative farming strategy, the subjective norm is:

$$
S N_{A}^{f}=\left\{\begin{array}{c}
0 \text { if }\left(I_{C}^{f}>T A\right) \\
\sum_{i=1}^{2}\left(W_{i}^{f}\left(\overline{P_{A, l}^{f}}-\frac{\sum_{v \neq f}^{N}\left(C_{v}^{f} P_{i}^{v}\right)}{\sum_{v \neq f}^{N} C_{v}^{f}}\right)\right) \text { otherwise }
\end{array}\right.
$$

If, in the opinion of other agents, especially those to whom agent $f$ lends great credibility ("important others") the alternative is not better, then it is judged not good enough to improve the bad situation. Agent $f$ will thus tend to stay with its current strategy. Otherwise, if the alternative is better, it is considered a useful option to improve the bad situation. Agent $f$ 's subjective norm will thus strengthen its intention to change to the alternative by increasing its satisfaction on the alternative strategy.

If an agent changes farming strategy, it will also change the importance given to each dimension of practice.

For the agents applying conventional strategy: $W_{0}=W(0,0) ; W_{l}=1-W(0,0)$ 
For the agents applying organic strategy: $W_{0}=1-W(0,0) ; W_{l}=W(0,0)$

\section{Credibility update}

The relationship between agents is characterized by the credibility one gives to another, which depends on an agent's personal view of its difference in practice to another agent. For agent $f$, its difference to agent $v$ is the sum of the difference for each dimension of practice weighted by the importance given to each dimension.

$$
D_{v}^{f}=\sum_{i=1}^{2}\left(W_{i}^{f}\left(P_{i}^{v}-P_{i}^{f}\right)\right)
$$

The credibility that agent $f$ gives to agent $v$ is calculated with $f$ 's difference to $v$ :

$$
C_{v}^{f}=\frac{1}{1+e^{-\alpha D_{v}^{f}}}
$$

Where $\alpha$ is the parameter used to characterize the slope of logistic function.

If agent $v$ 's practice is worse than agent $f$ 's, $f$ gives little credibility to $v$. As agent $v$ 's practice get better compared to $f$ 's, $f$ gives more credibility to $v$.

In Figure 2, the difference between $f$ and $v$ is plotted on the $\mathrm{x}$-axis. When this difference is negative, it means that $v$ has a worse practice than $f$, and $f$ gives little credibility to $v$. When the difference is positive, $v$ has a better practice than $f$, and $f$ gives greater credibility to $v$.

The lines with different colors represent the variation in different $\alpha . \alpha$ characterizes the degree of bias that an agent has for others with better practices. When $\alpha$ is small, the bias is small. An agent tends to give the same credibility to others, whether or not they have better practices. If $\alpha$ is big, the bias is strong. Only other agents with better practices are credible.

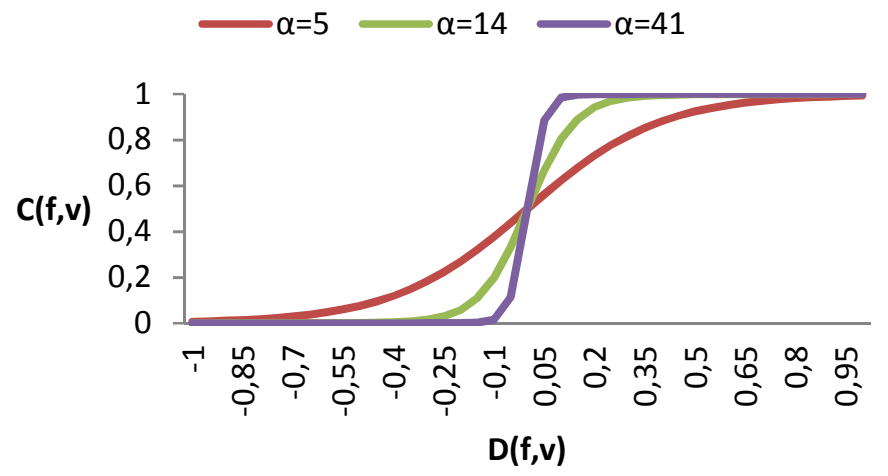

Figure 2-The credibility ( $y$-axis) agent $f$ gives to $\mathrm{v}$ depends on $\mathrm{f}$ 's difference ( $x$-axis) to $\mathrm{v}$ for three values of parameter $\alpha$ (different-colored lines)

In this model, every two agents are connected. As we know, credibility depends on an agent's perceived difference in practice to another. This credibility is then used to update the agent's practice which can change the perceived difference. Thus, the relationship associated to credibility is dynamic.

\section{Practice update}

In our model, practice is updated by comparing against others' practices. In particular, an agent's practice can be strongly influenced by the practice of more credible peers, i.e; "important others".

$$
\Delta P_{i}^{f}=\frac{\sum_{v \neq f}^{N} C_{v}^{f}\left(P_{i}^{v}-P_{i}^{f}\right)}{\sum_{v \neq f}^{N} C_{v}^{f}}
$$

Both dimensions of practice are between 0 and 1 . The productivity performance of a farm $f$ is also limited by its farmer's reference. 
Author-produced version of the article published in Natural Resource Modeling, 2018. The original publication is available at http://wwwsciencedirect.com/ doi : 10.1111/nrm.12171

$$
P_{0}^{f, t+1}=\left\{\begin{array}{c}
0 \text { if }\left(P_{0}^{f, t}+\Delta P_{0}^{f}<0\right) \\
R^{f} \text { if }\left(\left(P_{0}^{f, t}+\Delta P_{0}^{f}\right)>R^{f}\right) \\
P_{0}^{f, t}+\Delta P_{0}^{f} \text { otherwise }
\end{array}\right.
$$

Environmental performance is updated as follows:

$$
P_{1}^{f, t+1}=\left\{\begin{array}{l}
0 \text { if }\left(P_{1}^{f, t}+\Delta P_{1}^{f}<0\right) \\
1 \text { if }\left(\left(P_{1}^{f, t}+\Delta P_{1}^{f}\right)>1\right) \\
P_{1}^{f, t}+\Delta P_{1}^{f} \text { otherwise }
\end{array}\right.
$$

A special case: if agent $f$ looks for an alternative and cannot find a peer at $f$ 's level of production applying $f$ 's alternative strategy, it will look for an alternative in the media. If, after evaluation, agent $f$ adopts the alternative found in the media, then it will also copy the practice:

$$
P_{i}^{f}=P_{\text {model }, i}^{f}
$$

\section{Model behaviors}

The model aims to study why some agents do not change farming strategy. The number of agents having changed farming strategy during the simulation is measured to diagnose the dynamic behavior. This part of the paper studies the behaviors of the model considering the impact of the most determinant parameters.

Table 1 gives the definitions and values of the variables and parameters included in the model.

\begin{tabular}{|l|l|l|}
\hline Notation & Definition & Value(s) \\
\hline N & Number of agents in the population & 100 \\
\hline ITE & Number of iterations in the simulation & 100 \\
\hline M & Size of memory of agent's farming strategies and practices & 10 \\
\hline F0 & Proportion of small-farm farmers among all farmers & 0.3 \\
\hline F1 & Proportion of medium-farms farmers among all farmers & 0.5 \\
\hline E0 & Environmental performance of small conventional farms & 0.5 \\
\hline E1 & Environmental performance of medium conventional farms & 0.4 \\
\hline E2 & Environmental performance of large conventional farms & 0.2 \\
\hline$\alpha$ & Slope of the logistic function in the computation of credibility & {$[5,41]$} \\
\hline TA & Threshold to consider an alternative farming strategy & {$[0.4,0.5]$} \\
\hline TO & Threshold to change farming strategy & {$[0.01,0.1]$} \\
\hline Q0 & Level of production for small farms & {$[0.1,0.3]$} \\
\hline Q1 & Level of production for medium farms & {$[0.4,0.6]$} \\
\hline Q2 & Level of production for large farms & {$[0.7,0.9]$} \\
\hline W(0,0) & $\begin{array}{l}\text { Importance given to the dimension of practice corresponding to an agent's } \\
\text { farming strategy }\end{array}$ & {$[0.6,0.1]$} \\
\hline YLD & $\begin{array}{l}\text { Proportional decrease in productivity performance after the conversion to } \\
\text { organic farming strategy }\end{array}$ & {$[0.05,0.3]$} \\
\hline TD & $\begin{array}{l}\text { Threshold for the duration of confirmation and dissatisfaction before } \\
\text { changing farming strategy }\end{array}$ & $\{5,6, \ldots, 10\}$ \\
\hline
\end{tabular}

Table 1-Parameters and variables included in the model

Sobol sensitivity analysis was used to identify main parameters responsible for the proportion of agents having changed farming strategy at the end of simulation. See Appendix 1 for details. Sobol sensitivity analysis showed that the most important parameter is $T A$ (threshold to consider an alternative farming strategy), followed by $T O$ (threshold to change farming strategy), $W(0,0)$ (importance given to the dimension of practice corresponding to an agent's farming strategy) and $\alpha$ 
(slope of the logistic function in the computation credibility). In what follows, these important parameters are then varied while other less important ones are kept constant.

According to the hypothesis and simulation results of the model, $Q O$ (level of production for small farms) is considered to be $0.2, Q 1$ (level of production for medium farms) is $0.6, Q 2$ (level of production for large farms) is $0.9, T D$ (threshold for two durations in the change process) is 5 , and $Y L D$ (decrease of productivity performance after conversion) is 0.05 .

As stated in the "basic elements" part, satisfaction with current farming strategy is between 0 and 1 , with 0.5 the neutral satisfaction value. Satisfaction below 0.5 is considered dissatisfaction. Otherwise, the agent is satisfied. So, $T A$ is considered to be a value below 0.5 . We varied it from 0.41 to 0.49 in increments of 0.02 , since in simulation practice it is rare for satisfaction to go below 0.41 .

Change of strategy depends on how satisfaction compares between current and alternative farming strategy. Some changes are relatively easy to adopt while others can only be adopted when the alternative is clearly much better than current farming strategy. TO represents how much higher satisfaction with alternative farming strategy should be compared to satisfaction with the current one in order to adopt the alternative (i.e. to change farming strategy). We varied it from 0.01 to 0.09 in increments of 0.02 .

$\alpha$ characterizes the degree of an agent's bias in credibility given to others having better practices. If $\alpha$ is small, the agent tends to give a mid-range credibility (about 0.5 ) to everyone in the population. When $\alpha$ is big, the agent has a strong bias for others having better practices. To explore different biases, we varied $\alpha$ from 5 to 41 in increments of 9 .

The importance given to each dimension of practice represents personal values. The importance given to the dimension representing farming strategy, called $W(0,0)$ in what follows, was varied at initialization from 0.6 to 1 in increments of 0.1 . As we only have conventional farmers at initialization, $W(0,0)$ is the importance given to productivity performance whereas the importance given to environmental performance is the complementary to 1.30 replications computed for each parameter set.

\section{Parameters that influence an agent's decision}

Here we present parameters that have an important influence in prompting a change of farming strategy, and investigate various reasons for agents not changing farming strategy.

\section{Changes due to TA and TO}

Table 2 shows the average proportion of agents having changed farming strategy for 30 replications considering all variations of parameters $\alpha$ (slope of the logistic function in the computation of credibility) and $W(0,0)$ (importance given to the dimension of practice corresponding to an agent's farming strategy) for each value of $T O$ (threshold to change farming strategy) and $T A$ (threshold to consider an alternative farming strategy). The results show that the proportion of agents having changed farming strategy is smaller when $T A$ is smaller and $T O$ is larger.

The proportion of agents having changed farming strategy is lowest when $T A$ equals 0.41 and $T O$ equals 0.09 . ' $T A$ equals 0.41 ' means that agent is dissatisfied only when its satisfaction with current farming strategy is below 0.41 . 'TO equals 0.09 ' means that satisfaction with the alternative strategy has to be at least 0.09 better than satisfaction with the current strategy for an agent to change farming strategy. Both conditions are the strictest, so the proportion of conversion is the least in this situation.

The proportion of agents having changed farming strategy is highest when $T A$ equals 0.49 and $T O$ equals 0.01 . In this case, the agent is easily dissatisfied with its current farming strategy and only has to be a little more satisfied with the alternative to adopt it. 


\section{Changes due to $\alpha$ and $W(0,0)$}

Table 3 shows the results with $\alpha$ and $W(0,0)$. As expected, the length of the value distribution ( 0 to $0.074)$ shows that the results are less sensitive to $\alpha$ and $W(0,0)$ than to $T O$ and $T A$.

\begin{tabular}{|l|l|l|l|l|l|}
\hline \multicolumn{7}{|c|}{ Average proportion of change } \\
\hline TO TA & 0.41 & 0.43 & 0.45 & 0.47 & 0.49 \\
\hline 0.01 & 0.004 & 0.030 & 0.060 & 0.141 & 0.264 \\
\hline 0.03 & 0.002 & 0.014 & 0.029 & 0.056 & 0.107 \\
\hline 0.05 & 0.000 & 0.007 & 0.018 & 0.036 & 0.065 \\
\hline 0.07 & 0.000 & 0.005 & 0.014 & 0.025 & 0.046 \\
\hline 0.09 & 0.000 & 0.002 & 0.010 & 0.019 & 0.037 \\
\hline
\end{tabular}

Table 2-Proportion of changes for different values of $T O$ and $T A$ averaged over all the values given by the variations of parameters $\alpha$ and $W(0,0)$ and their related 30 replications

\begin{tabular}{|l|l|l|l|l|l|}
\hline \multicolumn{6}{|l|}{ Average proportion of change } \\
\hline $\mathrm{W}(0, \theta) \quad \alpha$ & 5 & 14 & 23 & 32 & 41 \\
\hline 0.6 & 0.063 & 0.035 & 0.061 & 0.062 & 0.074 \\
\hline 0.7 & 0.056 & 0.051 & 0.047 & 0.054 & 0.062 \\
\hline 0.8 & 0.062 & 0.032 & 0.030 & 0.030 & 0.030 \\
\hline 0.9 & 0.073 & 0.019 & 0.012 & 0.030 & 0.033 \\
\hline 1 & 0.065 & 0.011 & 0.000 & 0.000 & 0.000 \\
\hline
\end{tabular}

Table 3-Proportion of change for different values of $\mathrm{W}$ $(0,0)$ and $\alpha$ averaged over all the values given by the variations of parameters TA and TO and their related 30 replications

\section{Changes due to level of production}

Outside the important indicators that emerged through the Sobol sensitivity analysis, we find that proportion of agents having changed farming strategy also depends on the farm's level of production. Indeed, as shown in Figure 3, farms having changed strategy are mostly small and large farmsmedium farms convert the least. Level of production represents an agent's right or capacity to produce in terms of productivity performance. Thus farms with different levels of production may have different mechanisms driving their decisions on whether to change strategy.

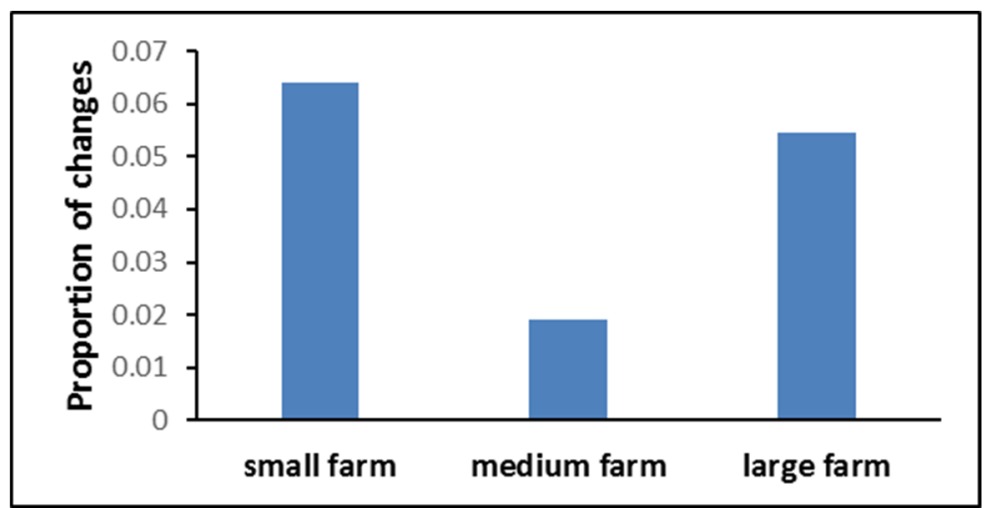

Figure 3-Average proportion of change according to type of farm (averaged over all the values given by the variations of parameters $T A, T O, W(0,0)$ and $\alpha$ and their related 30 replications)

Knowing this particular sensitivity to level of production that Sobol analysis failed to reveal, we now turn to further investigate the reasons for not changing farming strategy for each farm type.

\section{Different reasons not to convert for different farm types}

In our model, the computation of satisfaction is based on farming practices: an agent's own current practice, its practices in memory, and its perceived practices of other agents. Change of strategy is thus strongly related to the evolution of practices. The next subsections first give elements from an analytical study on the evolution of practices, then show the trajectories of "no conversion" in different cases, and lastly collect the corresponding reasons more or less related to evolution of practices.

\section{Evolution of farming practices: analytical study}

As shown in equations (19) to (21), an agent updates its practice by comparing against other agents' practices. If agents give the same credibility to others, then all agents in the population will converge to the same practice. Otherwise, if agents have strong bias for others with better practices, there will be several groups of practices. This is deduced from analysis of the dynamics of credibility. 


$$
C_{v}^{f}=\frac{1}{1+e^{-\alpha D_{v}^{f}}}=\left\{\begin{array}{c}
0.5 \text { if } \alpha=0 \text { or } D_{v}^{f}=0 \\
1 \text { if } \alpha \rightarrow \infty \text { and } D_{v}^{f}>0 \\
-1 \text { if } \alpha \rightarrow \infty \text { and } D_{v}^{f}<0
\end{array}\right.
$$

When $\alpha=0$, agent $f$ gives the same credibility (0.5) to all other agents.

$$
\begin{gathered}
\Delta P_{i}^{f}=\frac{\sum_{v \neq f}^{N} C_{v}^{f}\left(P_{i}^{v, t}-P_{i}^{f, t}\right)}{\sum_{v \neq f}^{N} C_{v}^{f}}=\frac{\sum_{v \neq f}^{N}\left(P_{i}^{v, t}-P_{i}^{f, t}\right)}{N} \\
P_{i}^{f, t}+\Delta P_{i}^{f}=P_{i}^{f, t}+\frac{\sum_{v \neq f}^{N}\left(P_{i}^{v, t}-P_{i}^{f, t}\right)}{N}=\frac{\sum_{v}^{N} P_{i}^{v, t}}{N}
\end{gathered}
$$

Agents try to have the averaged practice of the population. However, there are some agents that cannot reach it due to level-of-production limitations, as shown in equation (16). For example, small farms cannot have the productivity performance as the productivity performance initialized for medium farms because the maximum productivity performance of small farms is their level of production. However, medium and large farms can decrease their productivity performances. Consequently, small farms will keep nearly the same productivity performance whereas medium and large farms will have a lower productivity performance. After a certain period, all agents will convert to a same productivity performance that is the level of production of small farms.

Equation (17) shows that agents have no particular constraint in terms of environmental performance, so after a certain period, agents will converge to a same value for environmental performance.

Thus, when $\alpha=0$, agents give the same credibility to each other and convert to the same practice.

When $\alpha->\infty$, agents have strong bias for other agents with better practices and try to imitate their practices. This means that large farms cannot be influenced by medium and small farms. Medium farms try to imitate large farms' practices. In terms of productivity performance, medium farms can only reach their medium-farm level of production, which is lower than the productivity performance of large farms. However, in terms of environmental performance, medium farms will decrease it to be like large farms. Small farms are influenced by medium and large farms. With the same mechanism of updating practice as medium farms, small farms will keep their level of production as productivity performance, and decrease their environmental performance to be like large and medium farms.

$$
\begin{aligned}
& \Delta P_{i}^{f}=\frac{\sum_{v \neq f}^{N} C_{v}^{f}\left(P_{i}^{v, t}-P_{i}^{f, t}\right)}{\sum_{v \neq f}^{N} C_{v}^{f}}=\frac{\sum_{v \text { and } D_{v}^{f}>0}^{N}\left(P_{i}^{v, t}-P_{i}^{f, t}\right)}{N b\left(D_{v}^{f}>0\right)} \\
& P_{i}^{f, t}+\Delta P_{i}^{f}=P_{i}^{f, t}+\frac{\sum_{D_{v}^{f}>0}^{N}\left(P_{i}^{v, t}-P_{i}^{f, t}\right)}{N b\left(D_{v}^{f}>0\right)}=\frac{\sum_{D_{v}^{f}>0}^{N} P_{i}^{v, t}}{N b\left(D_{v}^{f}>0\right)}
\end{aligned}
$$

Thus, when $\alpha->\infty$ (large enough), agents have strong bias for other agents with better practices, so there will be several different sets of practice in the population due to level-of-production constraints.

Next we look at how evolutions of practices can explain the lack of farmers converting to organic.

\section{Different types of farms have different reasons and trajectories}

To further explain the reasons why an agent does not change, we studied agents' trajectories. For the sake of clarity, we consider a population of only 3 agents with 1 small farm, 1 medium farm, and 1 large farm. The simulations take 50 iterations. The results for each agent are analyzed with different variations of $\alpha$ and $W(0,0)$ and the maximum and minimum value of $T A$ and $T O$.

The other parameters are kept constant. All farmers are initialized as conventional farmers, and each farmer has a memory of its last 10 chosen strategies and practices $(M=10)$. The proportional decrease in productivity performance with the conversion to an organic farming strategy is $0.05(Y L D=0.05)$. 
The value for the delay mechanism $D$ is 5 . Level of production, initialized productivity performance and environmental performance for each type of farm are shown in Table 4.

\begin{tabular}{|l|l|l|l|l|}
\hline & $\begin{array}{l}\text { Level of } \\
\text { production }\end{array}$ & $\begin{array}{l}\text { Initialized productivity } \\
\text { performance }\end{array}$ & $\begin{array}{l}\text { Initialized environmental } \\
\text { performance }\end{array}$ & Synthesis \\
\hline Small farms & 0.2 & 0.2 & 0.5 & $Q 0=P_{0}=0.2, P_{l}=0.5$ \\
\hline Medium farms & 0.6 & 0.6 & 0.4 & $Q 1=P_{0}=0.6, P_{1}=0.4$ \\
\hline Large farms & 0.9 & 0.9 & 0.2 & $Q 2=P_{0}=0.9, P_{l}=0.2$ \\
\hline
\end{tabular}

Table 4-Initialized level of production, productivity performance and environmental performance by farm type

Overall, the analysis identified five reasons for non-conversion:

- An agent has never evaluated an alternative because it is always satisfied with its current strategy.

\section{(1. Current situation satisfactory)}

- An agent has evaluated an alternative:

- But after a certain period, it no longer considers the alternative

- Because subjective norm was the principle reason for its dissatisfaction, and the value of its subjective norm has increased (2. Dissatisfaction solved by subjective norm improvement)

- Because attitude was the principle reason for its dissatisfaction, and the value of its attitude has increased (3. Dissatisfaction solved by attitude improvement)

- It continues to evaluate the alternative, but the alternative is not good (4. Negative subjective norm of alternative)

- The alternative is much better than the current strategy, but the duration of dissatisfaction is not long enough when the reason was diagnosed. (5. Short dissatisfaction duration)

The related trajectories are described in further detail below.

Trajectories of the different farm types show reasons for non-conversion

In what follows, we present figures considering a 3-agent-population's evolution over time (from 1 to 10 iterations). Each agent has a different level of production. After the $10^{\text {th }}$ iteration, the result does not change.

Reason 1. Current situation satisfactory: an agent is socially favored by its peers and satisfied with current strategy

This is true for large and medium farms in Figure 4. Their satisfactions with current farming strategy $\left(I_{C}\right)$ are high enough, and higher than $T A$ (threshold to consider an alternative farming strategy, the value here is 0.41 ), to not consider an alternative. Conversely, the small farm is dissatisfied, as its $I_{C}$ is lower than $T A(0.41)$, but the computed satisfaction with the alternative farming strategy $\left(I_{A}\right)$ is not high enough to adopt it (i.e. $I_{A}-I_{C}$ is lower than $T O$ (threshold to change farming strategy, the value here is 0.01)). This difference satisfaction levels between the 3 agents is due to the difference in their subjective norms for the current farming strategy ( $S N_{C}$ in green) even though they share the same neutral attitude for current strategy $\left(A_{C}\right.$ in red).

The large farm has the highest productivity performance and is considered by other farms as having the best practice (because productivity performance is more important than environmental performance for conventional farmers), so the large farm is given the highest credibility and has the best subjective norm. Compared to the small farm, the medium farm has practice closer to the large farm, and is also given great credibility by the small farm, so it has a fairly good subjective norm. Conversely, the small farm has the lowest productivity performance, which is the worst practice in the population, so it is given poor credibility by others. It has a negative subjective norm. This makes it dissatisfied, and so it evaluates the alternative. 


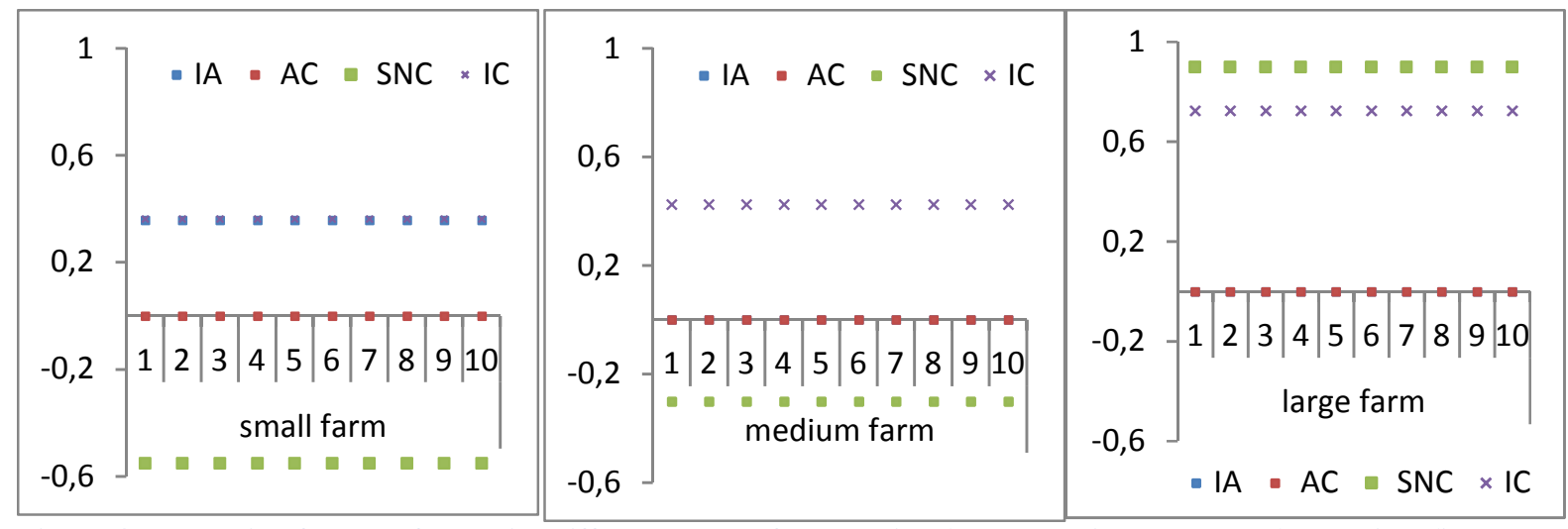

Figure 4-Evolution for each farm with different levels of production (large, medium and small) over time (in the $x$ axis above type of farm, $I_{A}$ is satisfaction with the alternative farming strategy, $A_{C}$ is attitude towards the current farming strategy, $S N_{C}$ is subjective norm for the current farming strategy and $I_{C}$ is satisfaction with the current farming strategy), $\alpha=41, T A=0.41, T O=0.01$, and $W(0,0)=1$.

So if a farmer who owns a medium or large farm benefits from a positive subjective norm regarding its current strategy, it can be socially satisfied enough. In this case, it does not even consider a change of farming strategy, and so does not evaluate the alternative.

Reason 2. Dissatisfaction solved by subjective norm improvement: an agent returns to being satisfied because of the convergence of agents' practices and the associated improvement of current subjective norm

This is true for the small farm in Figure 5. The small farm is at first dissatisfied and evaluates an alternative. The dissatisfaction does not last long enough to change farming strategy $(D D<T D)$. Later it returns to being satisfied and no longer considers an alternative. Note that the agent stops evaluating the alternative ( $I_{A}$ in dark blue) with a strong increase of subjective norm for current farming strategy $\left(S N_{C}\right.$ in green). This increase is due to the convergence of all agents' practices.

At first, the small-farm agent has a negative subjective norm because its productivity performance is the worst in the population. It tries to improve its practice by imitating the practices of its credible peers, particularly the large farm which is lower on environmental performance and higher on productivity performance. However, it can only decrease its environmental performance because its productivity performance is limited by level of production.

The medium farm can be influenced by the small farm and the large farm. On environmental performance, the small farm has a better result and the large farm has a worse result. Thus the medium farm does not change its environmental performance too much. On productivity performance, with the level-of-production constraint, the medium farm can only decrease its productivity performance to be like the small farm. The initialized practice of the medium farm is close enough to that of the large farm, so the large farm gives credibility to the medium farm and also updates its practice by looking at the medium farm's practice. Thus, the large farm decreases its productivity performance and increases its environmental performance. Finally, all agents converge to the same practice.

This convergence of practice reduces the practice differential between the small farm and the others. The small farm thus has a better subjective norm than before, which can make it return to being satisfied with current strategy.

In this case, with convergence of all agents' practices, an agent dissatisfied due to negative subjective norm can recover a neutral or positive subjective norm and become re-satisfied with current strategy. 


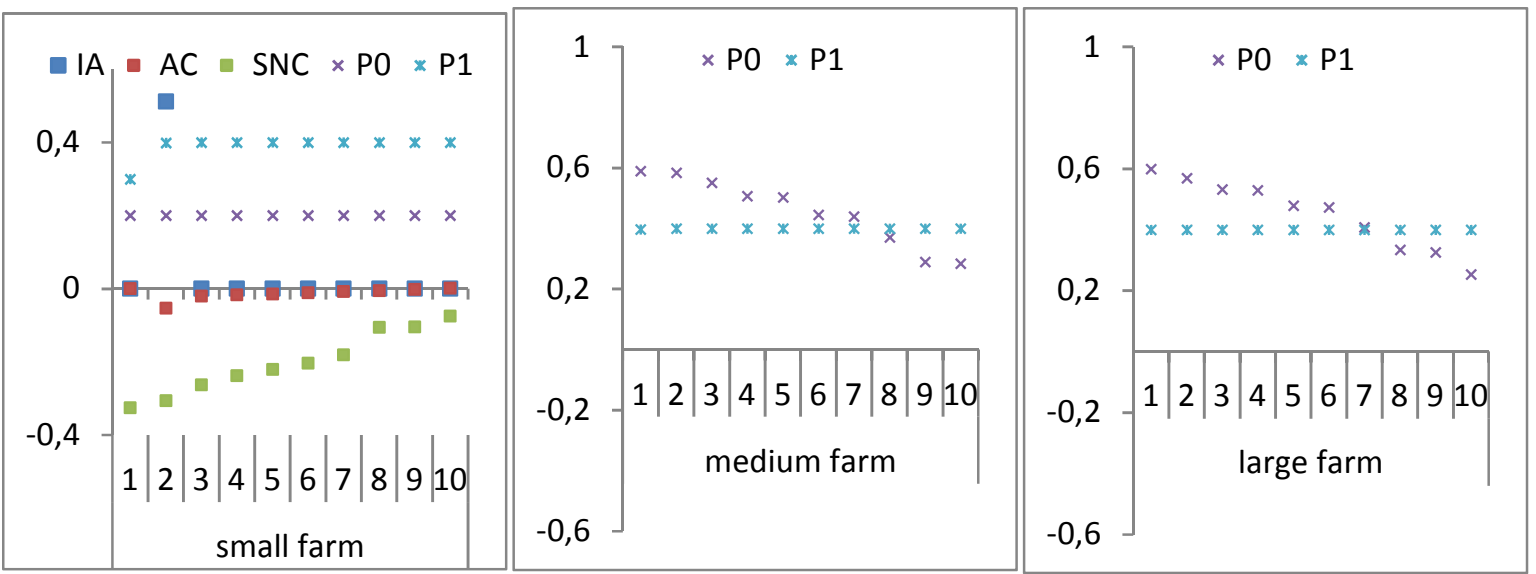

Figure 5-Evolution for each farm with different levels of production (large, medium and small) over time (in the $x$ axis above type of farm, $I A$ is satisfaction with the alternative farming strategy, $A C$ is attitude towards the current farming strategy, $S N C$ is subjective norm for the current farming strategy, $P 0$ is productivity performance 0 and $P 1$ is environmental performance), $\alpha=14, T A=0.41, T O=0.01$, and $W(0,0)=0.7$

In fact, this convergence of practice can arrive at many different levels according to different initializations of the population. When we change to a 10-agent population with 3 small farms, 5 medium farms and 2 large farms, the convergence of practice can encompass a part of the population instead of all agents.

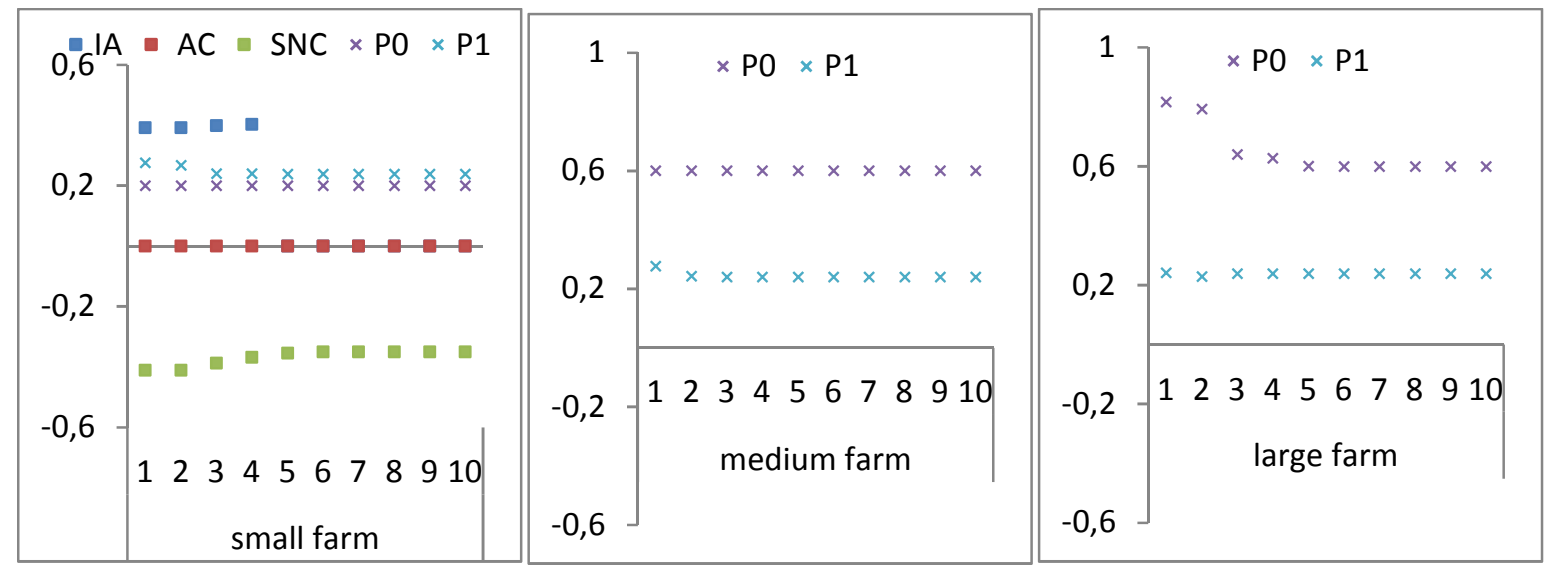

Figure 6-Evolution for each farm with different levels of production (large, medium and small) over time (in the $x$ axis above type of farm, $I A$ is satisfaction with the alternative farming strategy, $A C$ is attitude toward the current farming strategy, $S N C$ is subjective norm for the current farming strategy, $P 0$ is productivity performance and $P 1$ is environmental performance), $\alpha=14, T A=0.41, T O=0.09$, and $W(0,0)=1$

Figure 6 shows the trajectory for one agent from each level of production in a 10-agent population. Farms with the same level of production show the same pattern of behavior along the trajectory. As in the previous example presented in Figure 5, the small farm is dissatisfied at first and returns to being satisfied later and this change is again due to convergence of other agents' practices, but this time its own practice remains different from the practice of others. The medium farm converts to the large farm's practice, as in the previous example, but this time it is not influenced by the small farm. The large farm converts to the medium farm's practice as in the previous example.

In this example, the medium farm does not give credibility to the small farm so its practice is not influenced by the small farm, whereas in the previous example, the medium farm gave credibility to the small farm, which influenced its practice. Thus, the convergence is partial in this example and total in the previous example. This difference is due to different initializations of population.

So with the convergence of agents' practices, an agent can have a better subjective norm, which can bring it back to being satisfied with current strategy. This convergence can be partial or total with different initializations of population, and can change the practices of different farms in different ways. 
Reason 3. Dissatisfaction solved by attitude improvement: an agent returns to being satisfied because its practice is stabilized and its experience is updated

As shown in Figure 7, this reason is true for the large farm. For a short period, it is dissatisfied and evaluates an alternative. The dissatisfaction does not last long enough to change farming strategy $(D D$ $<T D$ ). Later it returns to being satisfied and no longer considers an alternative. The variation in its evaluation of alternative ( $I_{A}$ in dark blue) is strongly influenced by its attitude to current farming strategy $\left(A_{C}\right.$ in red). This change is because its practice is stabilized and its experience is updated.

With a small $\alpha$ equal to 5 , agents in this population tend to give nearly the same credibility to others regardless of whether their practices are better or not, so the convergence of practice happens quickly in this population. The small and the medium farms, being limited by level of production, cannot increase their productivity performance despite the large farm's influence. Thus, the large farm decreases its productivity performance a lot due to the credibility given to the medium and small farms. These farms with different levels of production are closer on environmental performance, so each agent can then freely update its environmental performance in a trade-off between credibility and environmental performance results.

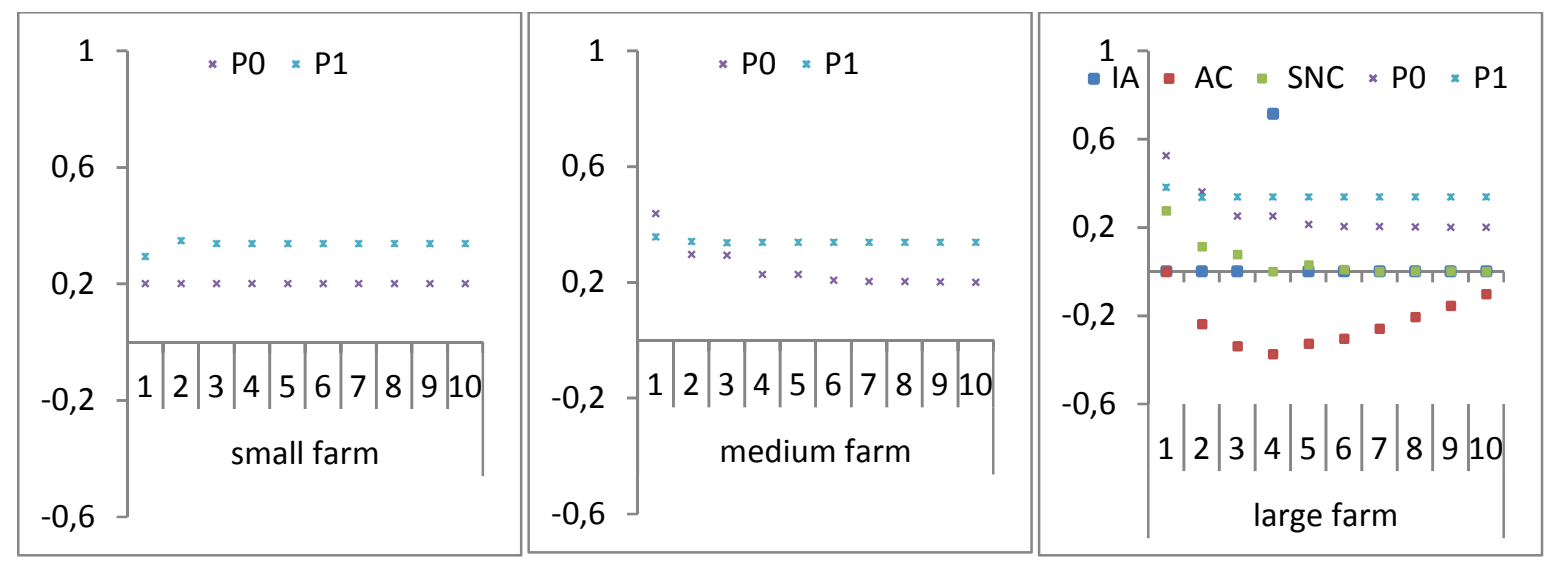

Figure 7-Evolution for each farm with different levels of production (large, medium and small) over time (in the $x$ axis above type of farm, $I A$ is satisfaction with the alternative farming strategy, $A C$ is attitude toward the current farming strategy, $S N C$ is subjective norm for the current farming strategy, $P O$ is productivity performance and $P 1$ is environmental performance), $\alpha=5, T A=0.41, T O=0.01$, and $W(0,0)=0.8$

Due to influence of other farms' productivity performance, the large farm experiences strong drop of productivity performance. This adds dissatisfaction to its attitude, which is the comparison between current practice and practices in memory. Its attitude toward the current strategy continues to deteriorate because its updated practice continues to fall lower compared to its experience. It keeps updating experience with new practices which are worse than before, and continues to have a negative attitude until the practices have converged and its experience stabilizes to reach a par with its current practice. If the time required to recover a neutral attitude is shorter than the threshold of dissatisfaction duration $(T D)$ before changing strategy $(D D<T D)$, the agent can return to being satisfied with its current strategy, just as shown in Figure 7.

In fact, if the speed of practice convergence, size of memory duration $(M)$, and threshold of duration before changing strategy $(T D)$ are different, we may have different outcomes.

Reason 4. Negative subjective norm of alternative: an agent keeps being dissatisfied and keeps evaluating the alternative which has a negative subjective norm

This reason is true for the small farm in Figure 8. The small farm keeps being dissatisfied and keeps evaluating the alternative. However, its satisfaction with the alternative $\left(I_{A}\right.$ in dark blue) does not change and is never good enough, i.e. less than the sum of its satisfaction with current farming strategy and threshold of changing farming strategy, IA $<I C+T O$ (threshold to change farming strategy)). 


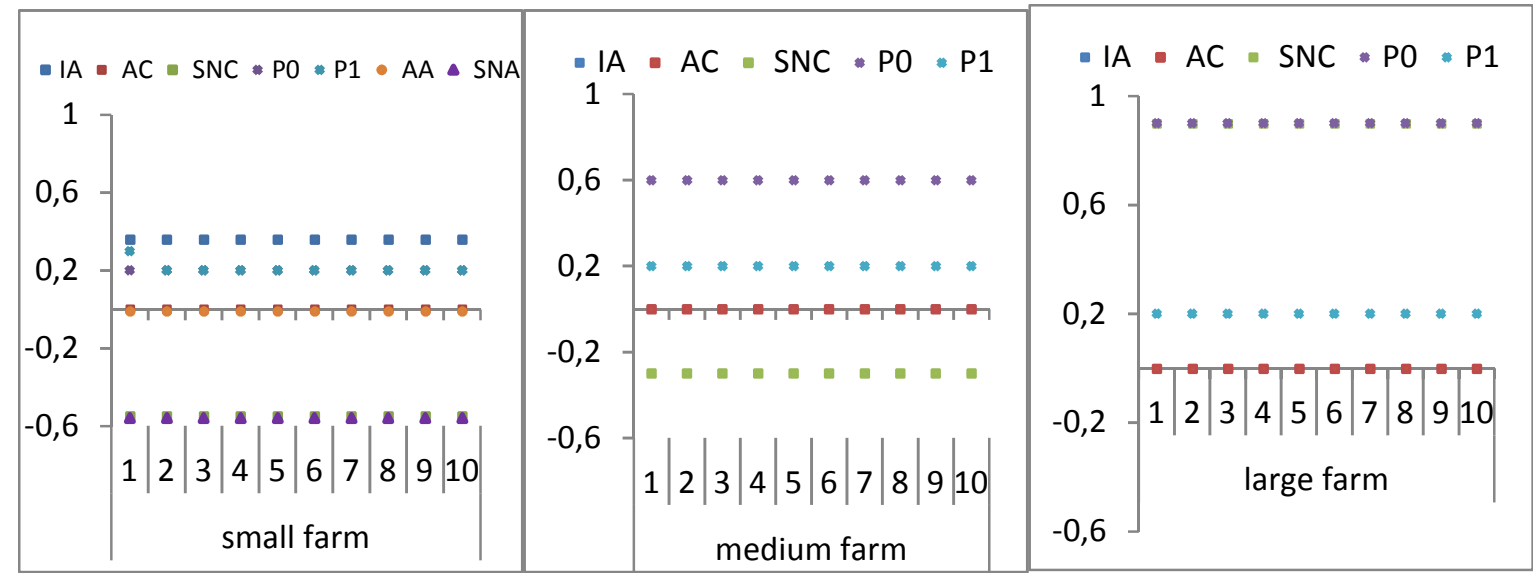

Figure 8-Evolution for each farm with different levels of production (large, medium and small) over time (in the $x$ axis above type of farm, $I A$ is satisfaction with the alternative farming strategy, $A C$ is attitude toward the current farming strategy, $S N C$ is subjective norm for the current farming strategy, $P 0$ is productivity performance, $P 1$ is environmental performance, $A A$ is attitude toward the alternative farming strategy and $S N A$ is subjective norm for the alternative farming strategy), $\alpha=23, T A=0.41, T O=0.01$, and $W(0,0)=1$

Conversely, the medium and large farms are satisfied with their current strategy. The difference is due to their subjective norm, because all farms have a same attitude ( $A_{C}$ in red). The small farm is dissatisfied because of its bad subjective norm $\left(S N_{C}\right.$ in green).

As the large farm has a bias for agents with better practices, it does not give credibility to medium and small farms. The medium farm does not give credibility to the small farm either. So as the small and the medium farms give high credibility to the large farm, they try to copy its practice. They decrease environmental performance to be like the large farm. On productivity performance, they cannot change because of their level-of-production constraint. Finally, all farms arrive at the same environmental performance ( $P_{1}$ in light green) and productivity performance ( $P_{0}$ in purple) corresponding to their respective levels of production.

Thus, the large farm is satisfied because it has the best practice among all farms. As they are all conventional farms, they all consider productivity performance as the most important. The medium farm is also satisfied because its productivity performance is similar to the large farm's and better than the small farm's.

The small farm has a negative social norm, and so it looks for an alternative. However, with the levelof-production constraint, the alternative also has a negative subjective norm $\left(S N_{A}\right.$ in dark purple) and is not good enough to trigger the change of strategy. The small farm thus stays dissatisfied and evaluates the alternative as not good enough to move for change.

An agent can keep its current farming strategy with dissatisfaction, because alternative is not good enough, particularly from a subjective norm point of view.

Reason 5. Short dissatisfaction duration: an agent does not change even though it has a good alternative, because it does not stay dissatisfied for long enough

Figure 9 shows the evolution of the large farm first presented in Figure 7. During certain iterations (e.g. iteration 4), the large farm is dissatisfied and evaluates the alternative. Its satisfaction with the alternative farming strategy is much higher than its satisfaction with current farming strategy (IA > $I C+T O)$. However, it does not change because it does not stay dissatisfied for long enough $(D D<T D)$. 


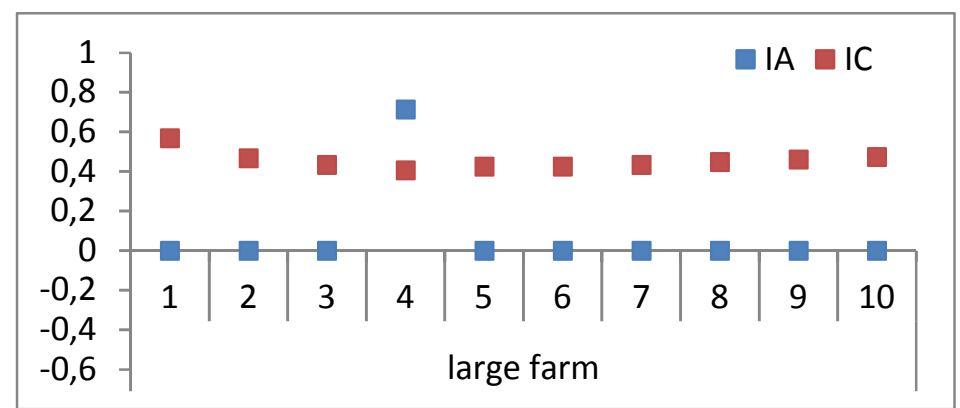

Figure 9-Evolution for the large farm over time (in the $x$-axis above type of farm, IA is satisfaction with alternative farming strategy, $I C$ is satisfaction with current farming strategy $), \alpha=5, T A=0.41, T O=0.01$, and $W(0,0)=0.8$.

So an agent can keep its current farming strategy with dissatisfaction despite having a good alternative, because it does not stay dissatisfied with the current strategy for long enough.

Overview of reasons for non-changing farming strategy on different types of farms

A detection algorithm is applied in the model to diagnose reasons for non-changing strategy (see Appendix 2). Table 5 shows the results of this automatic diagnosis for a 3-agent population with different levels of production (1 small, 1 medium, 1 large). The results present the most frequent reasons for non-changing farming strategy with different parameter sets, which are the combination of different values of $\alpha$ (slope of the logistic function in the computation of credibility) and $W(0,0)$ (importance given to the dimension of practice corresponding to the agent's farming strategy), and the maximum and the minimum values of $T O$ (threshold to change farming strategy) and $T A$ (threshold to consider an alternative farming strategy). Each number associated to a color in each cell corresponds to the most frequent reasons over 1000 replications. An empty cell means that the agent changes farming strategy in this parameter-set.

The results show that reason 1 is the most frequent reasons, followed by reason 4, reason 2 and reason 3. Reason 5 does not appear in these tables as it is the most tightly conditioned reason.

When the importance of productivity performance is not substantially higher than the importance of environmental performance (top of the top table), a small farm can be satisfied with current strategy and does not consider the alternative at all because it is socially favored by its peers - reason 1 (current situation satisfactory). For the medium and large farms, most of the time they keep their current strategy with reason 1 (current situation satisfactory), especially the large farm. As they have a higher productivity performance in the population, they usually have a good evaluation of current strategy, particularly from a subjective norm point of view. So most of the time, they do not change farming strategy due to current situation satisfactory.

Reason 4 (negative subjective norm of alternative) is also an important reason for small and medium farms. This reason can be true when $\alpha$ (slope of the logistic function in the computation of credibility) and $W(0,0)$ (importance given to the dimension of practice corresponding to the agent's farming strategy) are big (bottom-right corner of the top and middle tables). Large farms are not influenced by small and medium farms when $\alpha$ is big, which implies a strong bias for credible farms (farms with better practices), so the difference between farms remains large, especially on productivity performance to which these conventional farms give more importance. Thus small and medium farms remain dissatisfied with their current strategy while neither current nor alternative strategy are satisfactory (impact of $\alpha$ and $W(0,0)$ ).

Reason 2 (dissatisfaction solved by subjective norm improvement) is usually found for small farms in a population with strong convergence of practice (impact of $\alpha$ ). The small farm has a negative subjective norm because of the level-of-production constraint. However, its difference to other agents can decrease as practice in the population converges, which can improve its subjective norm and make it return to being satisfied with current strategy.

Reason 3 (dissatisfaction solved by attitude improvement) also relates to a convergence of practice that can make an agent feel dissatisfied because of its worse practice than in memory, which can 
Author-produced version of the article published in Natural Resource Modeling, 2018. The original publication is available at http://wwwsciencedirect.com/ doi : 10.1111/nrm.12171

provoke a negative attitude. However, with the update of experience and stabilization of practice, the agent can return to being satisfied.

\begin{tabular}{|c|c|c|c|c|c|c|c|c|c|c|c|}
\hline \multirow{2}{*}{\begin{tabular}{|l|} 
W00 $\quad \alpha$ \\
small farm
\end{tabular}} & \multirow{2}{*}{$\begin{array}{ll}\text { small farm } \\
T O \quad \text { TA }\end{array}$} & \multicolumn{2}{|c|}{5} & \multicolumn{2}{|c|}{14} & \multicolumn{2}{|c|}{23} & \multicolumn{2}{|c|}{32} & \multicolumn{2}{|c|}{41} \\
\hline & & 0.41 & 0.49 & 0.41 & 0.49 & 0.41 & 0.49 & 0.41 & 0.49 & 0.41 & 0.49 \\
\hline \multirow[b]{2}{*}{0.6} & 0.01 & 1 & & 1 & & 1 & & 1 & & 1 & \\
\hline & 0.09 & 1 & & 1 & & 1 & & 1 & & 1 & \\
\hline \multirow[b]{2}{*}{0.7} & 0.01 & 1 & & 1 & & & & & & & \\
\hline & 0.09 & 1 & & 1 & & & & & & & \\
\hline \multirow[b]{2}{*}{0.8} & 0.01 & 1 & & 2,3 & & & & & & & \\
\hline & 0.09 & 2 & 2 & 2,3 & 2 & 4 & 4 & 4 & 4 & 4 & 4 \\
\hline \multirow[b]{2}{*}{0.9} & 0.01 & 2 & & & & & & & & & \\
\hline & 0.09 & 2 & 2 & 3 & 2 & 4 & 4 & $\overline{4}$ & 4 & 4 & 4 \\
\hline \multirow[b]{2}{*}{1} & 0.01 & 2 & 2 & 4 & 2 & 4 & 4 & 4 & 4 & 4 & 4 \\
\hline & 0.09 & 2 & 2 & 4 & 2 & 4 & 4 & 4 & 4 & 4 & 4 \\
\hline \begin{tabular}{|ll} 
W00 & $\alpha$ \\
\end{tabular} & medium farm & \multicolumn{2}{|c|}{5} & \multicolumn{2}{|c|}{14} & \multicolumn{2}{|c|}{23} & \multicolumn{2}{|c|}{32} & \multicolumn{2}{|c|}{41} \\
\hline medium farm & TO $\quad$ TA & 0.41 & 0.49 & 0.41 & 0.49 & 0.41 & 0.49 & 0.41 & 0.49 & 0.41 & 0.49 \\
\hline \multirow[b]{2}{*}{0.6} & 0.01 & 1 & & 1 & & 1 & & 1 & & 1 & \\
\hline & 0.09 & 1 & & 1 & & 1 & & 1 & & 1 & \\
\hline \multirow[b]{2}{*}{0.7} & 0.01 & 1 & & 1 & & 1 & & 1 & & 1 & \\
\hline & 0.09 & 1 & & 1 & & 1 & & 1 & & 1 & \\
\hline \multirow[b]{2}{*}{0.8} & 0.01 & 1 & & 1 & & 1 & & 1 & & 1 & \\
\hline & 0.09 & 1 & & 1 & & 1 & 4 & 1 & 4 & 1 & 4 \\
\hline \multirow[b]{2}{*}{0.9} & 0.01 & 1 & & 1 & & 1 & 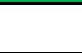 & 1 & & 1 & \\
\hline & 0.09 & 1 & & 1 & & 1 & 4 & 1 & 4 & 1 & 4 \\
\hline \multirow[b]{2}{*}{1} & 0.01 & 1 & & 1 & & 1 & 4 & 1 & 4 & 1 & 4 \\
\hline & 0.09 & 1 & & 1 & & 1 & 4 & 1 & 4 & 1 & 4 \\
\hline W00 $\quad \alpha$ & large farm & \multicolumn{2}{|c|}{5} & \multicolumn{2}{|c|}{14} & \multicolumn{2}{|c|}{23} & 3 & & 4 & \\
\hline large farm & TO $\quad$ TA & 0.41 & 0.49 & 0.41 & 0.49 & 0.41 & 0.49 & 0.41 & 0.49 & 0.41 & 0.49 \\
\hline & 0.01 & 1 & & 1 & & 1 & & 1 & & 1 & 1 \\
\hline 0.6 & 0.09 & 1 & & 1 & & 1 & & 1 & & 1 & 1 \\
\hline & 0.01 & 1 & & 1 & & 1 & & 1 & & 1 & 1 \\
\hline 0.7 & 0.09 & 1 & & 1 & & 1 & & 1 & & 1 & 1 \\
\hline & 0.01 & 1 & & 1 & & 1 & & 1 & & 1 & \\
\hline 0.8 & 0.09 & 1 & & 1 & & 1 & 1 & 1 & 1 & 1 & 1 \\
\hline & 0.01 & 2,3 & & 1 & & 1 & 1 & 1 & 1 & 1 & 1 \\
\hline 0.9 & 0.09 & 2,3 & & 1 & & 1 & 1 & 1 & 1 & 1 & 1 \\
\hline & 0.01 & 3 & & 1 & & 1 & 1 & 1 & 1 & 1 & 1 \\
\hline 1 & 0.09 & 3 & & 1 & & 1 & 1 & 1 & 1 & 1 & 1 \\
\hline
\end{tabular}

Tables 5-Different reasons for which farms do not change (from top to bottom: for small farms at top, medium farms at middle, and large farms at bottom). Reason 1 is current situation satisfactory; reason 2 is dissatisfaction solved by subjective norm improvement; reason 3 is dissatisfaction solved by attitude improvement; reason 4 is negative subjective norm of alternative; reason 5 is short dissatisfaction duration.

\section{Discussion}

The model proposes various possible farmer trajectories leading to the non-adoption of organic farming. It shows that the reason most farmers do not change strategy is because they are satisfied with their current situation. In some cases, farmers stay with their current strategy because they do not consider the alternative as a substantially better strategy than the current one. Sometimes, farmers 
dissatisfied with their current strategy become satisfied again because of an improvement of their personal evaluation or perceived evaluation by others of their current situation. In certain cases, they are sufficiently dissatisfied to evaluate the alternative which is interesting enough, but do not make to the decision to convert because they are not dissatisfied for long enough.

The discussion below looks at the value of each simulated reason for non-adoption of organic farming in light of the literature.

\section{The reason "alternative is badly evaluated"}

This reason corresponds to classical vision of farmer non-conversion. Most of the literature grounds non-conversion in features of organic farming (Kirchmann \& Thorvaldsson 2000; Edwards-Jones \& Howells 2001; Rigby \& Cáceres 2001; Trewavas 2001; Hill \& Lynchehaun 2002). The majority of conventional farmers interviewed in the Associatione ${ }^{2}$ project also argue that their reasons for nonconverting are related to features of organic farming, like market uncertainty or tough specifications.

In fact, farmers' concerns over converting to organic farming stem from their uncertainty over the future, which is consistent with an insufficient perceived behavioral control in TPA (Ajzen 1991). This could be an informative element to integrate in the next version of model.

This reason is the most frequent in the literature and in real-world interviews. However, it can result from deep introspection or just social opinion. Indeed, a farmer satisfied with his/her current situation almost never considers conversion (Deffuant et al, 2002), in which case his/her opinion on organic farming is more due to how he/she perceives the social norm, as in our model, than any deep introspection.

\section{The reason "satisfied with current strategy"}

As proposed by many researchers, a major change like the conversion to organic farming is always related to dissatisfaction with the current situation (Sutherland, Burton, et al. 2012; Chantre, Cerf \& Le Bail 2015; Huet et al. 2018), and ultimately a need for profound transformation (Barbier, Cerf \& Lusson 2015). Farmers who perceive the current situation as satisfactory do not make any big changes (Öhlmér, Olson \& Brehmer 1998).

In the model, this reason mainly applies for "high-production" farms (schematically called 'large farms' here). The stereotype of a farmer's main task is to produce more to feed the world. Farmers with large farms produce more than others and are considered as leaders of the system. Compared to others, especially lower-producing farms, they are more often satisfied with their current situation and do not consider changing. Their trajectories will be to continue with conventional farming and intensify more. This type of trajectory is also confirmed elsewhere (Xavier Coquil, Dedieu \& Béguin 2017; X. Coquil et al. 2013; Alavoine-Mornas \& Madelrieux 2014; Lamine et al. 2009). Bos, Smit \& Schröder (2013) and Groeneveld et al. (2016) also argue that large farms intensify more than small farms.

Conversely, farmers with a low level of production (small farms) are heavily burdened with production pressures and are more sensitive to external events, and so are more likely to be dissatisfied with the current situation. Thus, real small farms, but also our virtual small farms, are more likely to convert, as shown by the farmer interviews led under the Associatione ${ }^{2}$ project.

In the model, each farm has a fixed initial level of production without any external effect. In fact, farmers can increase/decrease their level of production by expanding/selling milking herd, etc. According to Dervillé et al. (2017), Barthélemy \& David (2001), and Gouin, (1988), levels of

\footnotetext{
${ }^{2}$ ASSOCIATIONE. Projet "Thématiques émergentes" [Emerging issues]. Auvergne-Rhône-Alpes regional council, 2015-2019
} 
production can be either transferred directly between farmers via land sales or exchanged through a departmental commission, either with or without compensatory payments. Over the last 20 years, farms have increased their overall level of production, as shown by Depeyrot (2017) for French dairy farms. This potential to increase, as well as actual effective increase, have meant that farmers have remained fairly satisfied with their current situation and delayed their change of strategy. A valuably informative next step in the modeling work would thus be to give virtual farmers the possibility to increase their level of production.

External events, such as price collapse or climate shock, are also essential drivers of farmers' strategy decisions. Contrary to Deffuant, Huet \& Weisbuch (2002), Kaufmann, Stagl \& Franks (2009) argue that economic factors are more influential than social factors in farmers' conversion decisions. Opportunities, especially market opportunities, are also considered as an important driver of transition by other authors (Barbier, Cerf \& Lusson 2015; Lamine et al. 2009; X. Coquil et al. 2013; MartinClouaire 2017; Chantre, Cerf \& Le Bail 2015; Alavoine-Mornas \& Madelrieux 2014). Thus external events, negative or positive, should be taken into account in future development of the model, since they can facilitate, or impede, the change.

\section{The reason "dissatisfaction solved by subjective norm improvement"}

As shown by Darré (1985) and the Associatione ${ }^{2}$ project interviews, farmers co-construct their practices, and most of them are now using less pesticides and other chemical inputs. Various studies provide useful insights into the roles of social evaluation (Barbier, Cerf \& Lusson 2015; Xavier Coquil, Dedieu \& Béguin 2017; Chantre, Cerf \& Le Bail 2015). To have a good enough social identity, a farmer in dissonance with his/her social group may change groups by changing strategy, or simply imitate the practice of other in-group members. This phenomenon also appears in our model: the convergence of farmers' practices sometimes prevents them from changing strategy. In such a case, the commonly adopted practice becomes a norm that dictates whether farmers are satisfied with themselves.

However, the complete convergence of practice shown in the model is quite rare in reality. As argued by Martin-Clouaire (2017), farmers always make decisions with neighbors or partners, even for daily routines, but their practices are rarely exactly duplicated. The complete convergence of practice in the model is due to the oversimplified initial distribution of farms' levels of production, as well as the impossibility of increasing level of production (as discussed in the previous subsection). Using our model to investigate initial populations built from real-world data, for example from the French census of farms, would be valuably informative here.

\section{The reason "dissatisfaction solved by attitude improvement"}

Earlier performance is very important for a farmer to evaluate his/her current situation (Öhlmér, Olson \& Brehmer 1998; Mintzberg, Raisinghani \& Theoret 1976; Wärneryd 1988). A farmer's decision on whether to convert can be strongly influenced by his/her experience. In addition, many authors consider learning from experience as an indispensable process of farming-system evolutions over the long term (Xavier Coquil, Dedieu \& Béguin 2017; Martin-Clouaire 2017; Xavier Coquil, Dedieu \& Beguin 2010). In the model, a farmer can have a better self-evaluation and return to being satisfied with his/her current situation by regularly integrating his/her experience and updating his/her personal attitude.

Similar to the reason "dissatisfaction solved by subjective norm improvement", updating attitude with experience can also lead to a progressive change of practice that, in turn, can lead the farmer to a more satisfactory social status.

Farmers learn with their experience, and this can usually reverse a change of their values, as shown by the importance given to each dimension of practice (Sutherland, Burton, et al. 2012; Xavier Coquil, Dedieu \& Béguin 2017). The dynamics of this importance warrant integration in future work. 


\section{The reason "short dissatisfaction duration"}

Mintzberg, Raisinghani \& Theoret (1976), Öhlmér, Olson \& Brehmer (1998) and Sutherland et al. (2012) argue that a major change in farming activities occurs only when there is an accumulation of experiences and events resulting in the farm manager recognizing and realizing a need for change. They also argue that the decision process needs to mature over a long period of time. Many converted farmers interviewed in the Associatione ${ }^{2}$ project also declared that they waited long after a sociotechnical diagnostic of the conversion impact before actually completing the final conversion. Alavoine-Mornas \& Madelrieux (2014) also observed this type of trajectory where some farmers wait for a long time to convert to organic farming even though everything is ready.

In order to understand long-term processes of change, some agronomists argue that professional transition processes such as the conversion to organic farming reflect the dichotomies not only between the farmer's wishes for the future and what proves possible, but also between problems that arise and the solutions that can be implemented (Martin-Clouaire 2017; Chantre, Cerf \& Le Bail 2015; Xavier Coquil, Dedieu \& Béguin 2017). Going organic can thus prove a slow and deliberative process that may take a really long time. However, different people will embrace change at a different pace. Some are more likely to be innovators than others. Some are risk-adverse and prefer being confirmed by the observation of others' lasting successes, as well as by their own experiments, thus making incremental changes (Hamal \& Anderson 1982; Girdžiūte 2012). Most farmers who experiment and progressively change their practices becomes quasi-organic farmers without deciding to convert (Sutherland 2011; Alavoine-Mornas \& Madelrieux 2014).

\section{Conclusions}

We built a dynamic version of the Theory of Reasoned Action to investigate how socio-psychological factors can explain why French dairy farmers in mountainous areas are not adopting organic farming practices despite the profitable economic opportunities involved. Our individual-based model defines a farmer's current or potential satisfaction regarding a farming strategy. We found that in most cases, a farmer with a large or medium farm in terms of level of production does not change strategy because he/she is satisfied with his/her current situation. The evaluation of the conversion to organic farming as an alternative is a highly influential factor in agents' decisions to go organic, especially for small farms. In many cases, even with the conversion to organic farming, small farms cannot improve their situation in terms of productivity performance compared to others, and so do not convert. In other cases, not being dissatisfied with the current situation for long enough is also a barrier to conversion. The model reveals that a farmer dissatisfied with his/her strategy can return to being satisfied if his/her personal attitude or subjective norm improve. Farmers continuously adapt their practices with peers to build their own collective response to external changes. Due to interaction between farmers, there are some progressive changes of practice that impede major change, and others that facilitate it. The change of farming practices appears central to understanding organic adoption, and thus warrants further research and modeling scholarship. It has to be considered not only at farm level but also at the population-of-farmers level. In addition, more research is needed on farmers' trajectories, as a major change can be effectuated via progressive minor changes or by a sudden rupture. These processes are complex and driven by interactions between social, individual and external factors. Further research on farmers' decision-making processes will help gain further insights into their decision-making environment and constraints.

\section{Acknowledgements}

We thank the Slovak Academy of Sciences, particularly Peter Kaufmann and Daniel W. Franks, for providing us with the IDARI survey data.

We thank the Associatione project and Auvergne-Rhône-Alpes regional council for providing us with the interviews with dairy farmers in "Pilats". 
Author-produced version of the article published in Natural Resource Modeling, 2018. The original publication is available at http://wwwsciencedirect.com/ doi : 10.1111/nrm.12171

\section{References}

Ajzen I. 1991. The Theory of Planned Behavior. Organizational Behavior and Human Decision Processes 50 (2): 179-211.

Ajzen I \& Fishbein M. 1975. Belief, Attitude, Intention and Behavior: An Introduction to Theory and Research. Reading, MA: Addison-Wesley.

Alavoine-Mornas F \& Madelrieux S. 2014. Passages à l'agriculture biologique. Une diversité de processus. Économie rurale. Agricultures, alimentations, territoires. 339-340: 65-79.

Armitage CJ \& Conner M. 2001. Efficacy of the Theory of Planned Behaviour: a meta-analytic review. British Journal of Social Psychology 40 (4): 471-499.

Barbier C, Cerf M \& Lusson JM. 2015. Cours de vie d'agriculteurs allant vers l'économie en intrants: Les plaisirs associés aux changements de pratiques. Activités 12 (12-2).

Barthélemy D \& David J. 2001. Production Rights in European Agriculture. Elsevier.

Beedell JDC \& Rehman T. 1999. Explaining farmers' conservation behaviour: Why do farmers behave the way they do? Journal of Environmental Management 57 (3): 165-76.

Beedell J \& Rehman T. 2000. Using social-psychology models to understand farmers' conservation behaviour. Journal of Rural Studies 16 (1): 117-127.

Boisdon I, Ott AS, Fourdin S \& Dockès A-C. 2013. "Typologie des trajectoires de conversion à l'agriculture biologique d'exploitations laitières de montagne. Rencontres Autour Des Recherches Sur Les Ruminants 20.

Boldero J. 1995. The prediction of household recycling of newspapers: The role of attitudes, intentions, and situational factors. Journal of Applied Social Psychology 25 (5): 440-62.

Bos J, Smit A \& Schröder JJ. 2013. "Is agricultural intensification in The Netherlands running up to its limits?" NJAS - Wageningen Journal of Life Sciences 66: 65-73.

Brown C, Alexander P, Holzhauer S \& Rounsevell MDA. 2017. Behavioral models of climate change adaptation and mitigation in land-based sectors. Wiley Interdisciplinary Reviews: Climate Change 8 (2).

Bui S, Cardona A, Lamine C \& Cerf M. 2016. Sustainability transitions: Insights on processes of niche-regime interaction and regime reconfiguration in agri-food systems. Journal of Rural Studies 48: 92-103.

Chantre E, Cerf M \& Le Bail M. 2015. Transitional pathways towards input reduction on French field crop farms. International Journal of Agricultural Sustainability 13 (1): 69-86.

Coello Coello CA, Lamont GB \& Van Veldhuizen DA. 2007. Evolutionary Algorithms for Solving Multi-Objective Problems. Vol. 5. Springer.

Coquil X, Lusson JM, Beguin B \& Dedieu B. 2013. Itinéraires vers des systèmes autonomes et économes en intrants: motivations, transition, apprentissages. Rencontres Recherches Ruminants 20.

Coquil X, Dedieu B \& Beguin P. 2010. How do livestock and crop sciences represent evolutions of farming systems? A review. 9th International Farming Systems Association.

Coquil X, Dedieu B \& Beguin P. 2017. "Professional transitions towards sustainable farming systems: The development of farmers' professional worlds. Work 57 (3): 325-337.

Darnhofer I, Schneeberger W \& Freyer B. 2005. Converting or not converting to organic farming in Austria: Farmer types and their rationale. Agriculture and Human Values 22 (1): 39-52.

Darré J-P. 1985. La Parole et La Technique: L'univers de Pensée Des Éleveurs Du Ternois. Vol. 10. Editions L'Harmattan.

Dedieu M-S, Lorge A, Louveau O \& Marcus V. 2017. "Les Exploitations En Agriculture Biologique: Quelles Performances Économiques?".

Deffuant G, Amblard F, Weisbuch G \& Faure T. 2002. How can extremism prevail? A study based on the relative agreement interaction model. Journal of Artificial Societies and Social Simulation $5(4)$.

Deffuant G, Carletti T \& Huet S. 2012. The Leviathan Model: absolute dominance, generalised distrust, small worlds and other patterns emerging from combining vanity with opinion propagation. The Journal of Artificial Societies and Social Simulation 16 (1).

Deffuant G, HuetS \& Amblard F. 2005. An individual - based model of innovation diffusion mixing social value and individual benefit. American Journal of Sociology 110 (4): 1041-69. 
Deffuant G, Huet S \& Weisbuch G. 2002. Agent Based Simulation of Organic Farming Conversion in Allier Département. Edward Elgar Publishers.

Depeyrot J-N. 2017. "Les Transformations Du Paysage Laitier Français Avant La Sortie Des Quotas."

Dervillé M, Allaire G, Maigné E \& Cahuzac E. 2017. Internal and contextual drivers of dairy restructuring: Evidence from French mountainous areas and post-quota prospects. Agricultural Economics 48 (1): 91-103.

Dockès A-C, Boisdon I, Experton C \& Fourdin S. 2013. Mise au point de méthodes et outils innovants pour développer l'élevage laitier biologique en zones de Piémont et montagne. Innovations Agronomiques 32: 243-57.

Dollé J-B, Delaby L, Plantureux S, Moreau S, Amiaud B, Charpiot A, Manneville V, Chanseaume A, Chambaut H \& Le Gall A. 2013. Impact environnemental des systèmes bovins laitiers français. INRA Prod. Anim 26 (2): 207-220.

Dowd A-M, Marshall N, Fleming A, Jakku E, Gaillard E \& Howden M. 2014. The role of networks in transforming Australian agriculture. Nature Climate Change 4 (7): 558-63.

Edwards-Jones G \& Howells O. 2001. The origin and hazard of inputs to crop protection in organic farming systems: Are they sustainable?" Agricultural Systems 67 (1): 31-47.

Fairweather JR. 1999. Understanding how farmers choose between organic and conventional production: Results from New Zealand and policy implications. Agriculture and Human Values 16 (1): 51-63.

Fairweather JR, Hunt LM, Rosin CJ \& Campbell HR. 2009. Are conventional farmers conventional? Analysis of the environmental orientations of conventional New Zealand farmers. Rural Sociology 74 (3): 430-454.

Fielding KS, Terry DJ, Masser BM \& Hogg MA. 2008. Integrating social identity theory and the theory of planned behaviour to explain decisions to engage in sustainable agricultural practices. British Journal of Social Psychology 47 (1): 23-48.

Fishbein M. 1980. "A theory of reasoned action: Some applications and implications. Nebraska Symposium on Motivation 27: 65-116.

Gardebroek C. 2002. Farm-specific factors affecting the choice between conventional and organic dairy farming. EAAE 2002 International Congress, Zaragoza (Spain) 28: 31.

Girdžiūtè L. 2012. Risks in agriculture and opportunities of their integrated evaluation. Procedia Social and Behavioral Sciences, World Conference on Business, Economics and Management (BEM-2012), May 4-6 2012, Antalya, Turkey, 62 (Supplement C): 783-90.

Goldstone RL \& Janssen MA. 2005. Computational models of collective behavior. Trends in Cognitive Sciences 9 (9): 424-30.

Gouin DM. 1988. "Peut-on se passer d'un marché des quotas? Cahiers d'Economie et Sociologie Rurales 7 (2): 179-192.

Grimm V. 1999. "Ten years of individual-based modelling in ecology: What have we Learned and what could we learn in the future? Ecological Modelling 115 (2): 129-48.

Groeneveld A, Peerlings J, Bakker M \& Heijman W. 2016. The effect of milk quota abolishment on farm intensity: shifts and stability." Wageningen Journal of Life Sciences 77 (Suppl.C): 2537.

Hamal KB \& Anderson JR. 1982. A note on decreasing absolute risk aversion among farmers in Nepal. Australian Journal of Agricultural Economics 26 (3): 220-25.

Hansson H, Ferguson R \& Olofsson C. 2012. Psychological constructs underlying farmers' decisions to diversify or specialise their businesses - An application of theory of planned behaviour. Journal of Agricultural Economics 63 (2): 465-82.

Hill H \& Lynchehaun F. 2002. Organic milk: Attitudes and consumption patterns. British Food Journal 104 (7): 526-42.

Huet S \& Deffuant G. 2015. The Leviathan model without gossips and vanity: The richness of influence based on perceived hierarchy. In Social Simulation Conference 2015, 13-p.

Huet S, Rigolot C, Xu Q, de Cacqueray-Valmenier Y \& Boisdon I. 2018. Toward modelling of transformational change processes in farm decision-making. Agricultural Sciences.

Janssen S \& van Ittersum MK. 2007. Assessing farm innovations and responses to policies: A review of bio-economic farm models. Agricultural Systems 94 (3): 622-36. 
Kaufmann P, Stagl S \& Franks DW. 2009. Simulating the diffusion of organic farming practices in two new EU member states. Ecological Economics 68 (10): 2580-93.

Kirchmann H \& Thorvaldsson G. 2000. Challenging targets for future agriculture. European Journal of Agronomy 12 (3): 145-161.

Lamine C \& Bellon S. 2009. Conversion to organic farming: A multidimensional research object at the crossroads of agricultural and social sciences. A review. Agronomy for Sustainable Development 29 (1): 97-112.

Lamine C, Meynard J-M, Perrot N \& Bellon S. 2009. Analyse des formes de transition vers des agricultures plus écologiques: Les cas de l'agriculture biologique et de la protection intégrée. Innovations Agronomiques 4: 483-93.

Louhichi K, Alary V \& Grimaud P. 2004. A dynamic model to analyse the bio-technical and socioeconomic interactions in dairy farming systems on Réunion Island. Animal Research 53 (5): 363-82.

Lynne GD, Casey CF, Hodges A \& Rahmani M. 1995. Conservation technology adoption decisions and the theory of planned behavior. Journal of Economic Psychology 16 (4): 581-98.

Martin-Clouaire R. 2017. Modelling operational decision-making in agriculture. Agricultural Sciences 8 (7): 527-544.

Mason WA, Conrey FR \& Smith ER. 2007. Situating social influence processes: dynamic, multidirectional flows of influence within social networks. Personality and Social Psychology Review 11 (3): 279-300.

Michelsen J. 2001. Organic farming in a regulatory perspective. The Danish case. Sociologia Ruralis 41 (1): 62-84.

Mintzberg H, Raisinghani D \& Theoret A. 1976. The structure of 'unstructured' decision processes. Administrative Science Quarterly 21 (2): 246.

Öhlmér B, Olson K \& Brehmer B. 1998. Understanding farmers' decision making processes and improving managerial assistance. Agricultural Economics 18 (3): 273-90.

Olabisi LS, Qi Wang R \& Ligmann-Zielinska A. 2015. Why don't more farmers go organic? Using a stakeholder-informed exploratory agent-based model to represent the dynamics of farming practices in the Philippines. Land 4 (4): 979-1002.

Pavie J, Dockès A-C \& Echevarria L. 2002. Etude des freins à la conversion à l'agriculture biologique des exploitations laitières bovines. Institut de L'Élevage.

Rickards L \& Howden SM. 2012. Transformational adaptation: agriculture and climate change. Crop and Pasture Science 63 (3): 240.

Rigby D \& Cáceres D. 2001. Organic farming and the sustainability of agricultural systems. Agricultural Systems 68 (1): 21-40.

Rigby D, Young T \& Burton M. 2001. The development of and prospects for organic farming in the UK. Food Policy 26: 599-613.

Robert M, Thomas A \& Bergez J-E. 2016. Processes of adaptation in farm decision-making models. A review. Agronomy for Sustainable Development 36 (4): 64.

Rogers EM. 1983. Diffusion of Innovations. 3rd ed. New York : London: Free Press ; Collier Macmillan.

Sainte-Beuve J, Bougherara D \& Latruffe L. 2011. Performance économique des exploitations biologiques et conventionnelles: Levier économique à la conversion." In: Les Transversalités de l'agriculture Biologique, Strasbourg, 10pp.

Saltelli A, 2008. Global Sensitivity Analysis: The Primer. Chichester, England; Hoboken, NJ: John Wiley.

Schneeberger W, Darnhofer I \& Eder M. 2002. Barriers to the adoption of organic farming by cashcrop producers in Austria. American Journal of Alternative Agriculture 17 (01): 24-31.

Sobol IM. 1993. Sensitivity estimates for nonlinear mathematical models. Mathematical Modelling and Computational Experiments 1 (4): 407-414.

Sutherland L-A. 2011. 'Effectively Organic': environmental gains on conventional farms through the market? Land Use Policy 28 (4): 815-24.

Sutherland L-A, Burton RJF, Ingram J, Blackstock K, SleeB \& Gotts N. 2012. Triggering change: towards a conceptualisation of major change processes in farm decision-making. Journal of Environmental Management 104: 142-51. 
Author-produced version of the article published in Natural Resource Modeling, 2018. The original publication is available at http://wwwsciencedirect.com/ doi : 10.1111/nrm.12171

Sutherland L-A, Gabriel D, Hathaway-Jenkins L, Pascual U, Schmutz U, Rigby D, Godwin R, et al. 2012. The 'Neighbourhood Effect': a multidisciplinary assessment of the case for farmer coordination in agri-environmental programmes. Land Use Policy 29 (3): 502-12.

Tovey H. 1997. Food, environmentalism and rural sociology: On the organic farming movement in Ireland. Sociologia Ruralis 37 (1): 21-37.

Trewavas A. 2001. Urban myths of organic farming. Nature 410 (6827): 409.

Wärneryd KE. 1988. Social influence on economic behavior. In: van Raaij WF, van Veldhoven GM, Wärneryd KE. (eds) Handbook of Economic Psychology. Springer, Dordrecht.

Zhang X-Y, Trame MN, Lesko LJ \& Schmidt S. 2015. Sobol sensitivity analysis: a tool to guide the development and evaluation of systems pharmacology models CPT Pharmacometrics \& Systems Pharmacology 4 (2): 69-79. 


\section{Appendix 1. Sobol sensitivity analysis to identify the main parameters for the proportion of agents changing farming strategy}

The model aims to study why some agents do not convert. The number of agents having changed their farming strategies during the simulation is measured to diagnose their dynamic behavior.

In the model, some parameters are considered as constant. However, the model still has 9 parameters to vary. Having so many parameters in the model makes it difficult to analyze how all these parameters can influence our results. We thus we started with a global sensitivity analysis aiming to evaluate the extent to which changes in the model parameters will affect model output (Zhang et al. 2015). We opted for a Sobol analysis (Sobol 1993). This method uses straightforward Monte-Carlo integration of multidimensional integrals to compute ANOVA-like decompositions of the output variance, as well as the main effects, the interaction terms and the higher-order terms. It is implemented using the MultiObjective Evolutionary Algorithm (MOEA) framework in Java. The MOEA framework is a library of multiple-objective evolutionary optimization algorithms, with Sobol sensitivity analysis being one of the optimization libraries (Coello, Lamont \& Van Veldhuizen 2007; Saltelli 2008)

\section{Experimental design}

\section{Initialization}

As presented in the model description, level of production can be a synthetic indicator that represents farm characteristics like farm size or herd number ... In the model, we consider 3 different levels of production, which correspond to three types of farm: small, medium and large.

Based on analysis of interviews and experts' arguments, for the conventional farms, we assume that the larger the farm, the stronger its intensification and the worse its environmental performance (Dolle et al. 2013). So at initialization, the farms with a low level of production have the best environmental performance and the farms with a high level of production have the worst environmental performance.

As we know, an agent lends more importance to the dimension that represents its farming strategy (conventional farming strategy: productivity performance; organic farming strategy: environmental performance). The sum of the importance given to these two dimensions is 1 . So at initialization, the importance given to the dimension defining the farming strategy is above 0.5 . The exact value depends on the initialization. In the model, this is a parameter that varies between 0.5 and 1 .

\section{Constant parameters}

To simplify the analysis of agents' dynamic behaviors, the following parameters are kept constant.

- $\quad N$ : the number of agents in the population, takes the value 100 ;

- ITE: the number of iterations, takes the value 100;

- $M$ : the size of the memory of agent's farming strategies and practices, takes the value 10 ;

- $F 0$ : the proportion of small-farm farmers among all farmers at initialization, takes the value 0.3 ;

- F1: the proportion of medium-farm farmers among all farmers at initialization, takes the value 0.5;

- EO: the environmental performance of small conventional farms, takes the value 0.5;

- E1: the environmental performance of medium conventional farms, takes the value 0.4 ;

- E2: the environmental performance of large conventional farms, takes the value 0.2.

Note that there are no organic farmers in the population at initialization. We assume that organic farmers have to emerge from the dynamics.

\section{Parameters which vary}

We decided to vary the following parameters to study the model's dynamics, with a focus on the decision-making function based on the theory of reasoned action:

- $\alpha$ : slope of the logistic function in the computation of credibility, takes a value between 5 and 41 ; 
- $\quad T A$ : the threshold to consider an alternative farming strategy, takes a value between 0.4 and 0.5 ;

- $T O$ : the threshold to change farming strategy, takes a value between 0.01 and 0.1 ;

- $Q 0$ : the level of production for small farms, takes a value between 0.1 and 0.3 ;

- Q1: the level of production for medium farms, takes a value between 0.4 and 0.6;

- $\quad$ Q2: the level of production for large farms, takes a value between 0.7 and 0.9 ;

- $W(0,0)$ : the initialized importance for the dimension of practice corresponding to an agent's farming strategy, takes a value between 0.6 and 1 ;

- $Y L D$ : the proportional decrease in productivity performance after the conversion to organic farming strategy, takes a value between 0.05 and 0.3 ;

- $T D$ : the threshold for the duration of confirmation and of dissatisfaction before changing farming strategy, takes a value between 5 and 10 .

\section{Sobol analysis parameter}

We generate 10000 initial samples from the pseudorandom Sobol sequence. There are 9 parameters that vary, and the number of total parameter-sets considered for the sensitivity analysis is $2 * 10,000 *(9+1)=200,000$.

\section{Results of the Sobol test}

Figure A1 shows the result of the sensitivity analysis for the number of agents having changed their farming strategies during the simulation. The results indicate that parameter $T A$ is the most important parameter, contributing to nearly $80 \%$ of the model output variability related to the number of agents having changed farming strategy at the end of the simulation. It is followed by the important parameters $T O, W(0,0)$ and $\alpha$. Error bars in the figure represent the bootstrap confidence intervals (1.96*standard error).

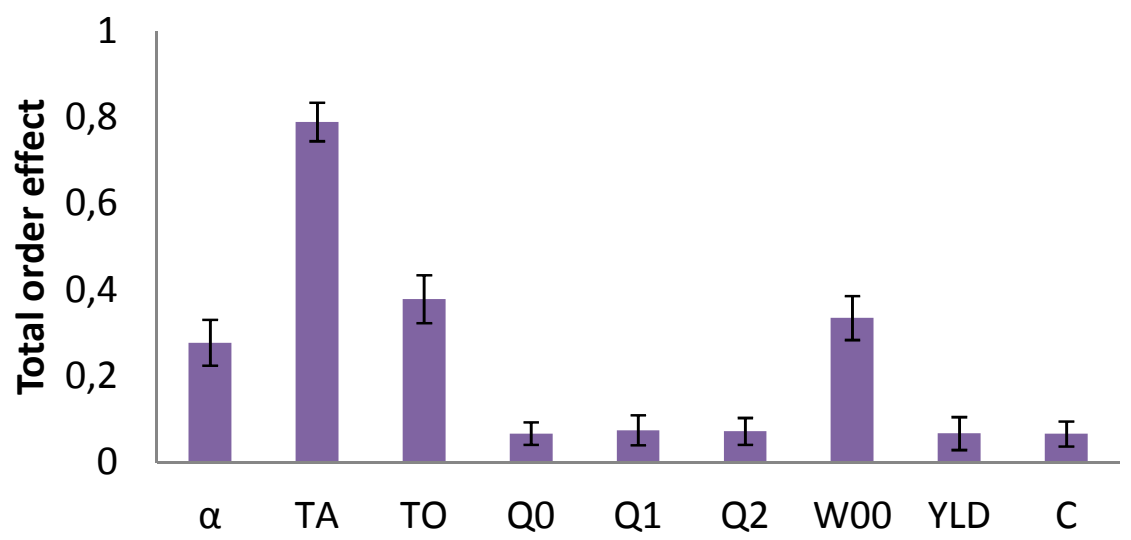

Figure A1-Results of the Sobol analysis. TA, TO, W $(0,0)$ and $\alpha$ are the most determinant parameters

We can thus focus on these four important parameters to deeply study the dynamics of our model. 
Author-produced version of the article published in Natural Resource Modeling, 2018. The original publication is available at http://wwwsciencedirect.com/ doi : 10.1111/nrm.12171

\section{Appendix 2. Reason for not changing detection algorithm}

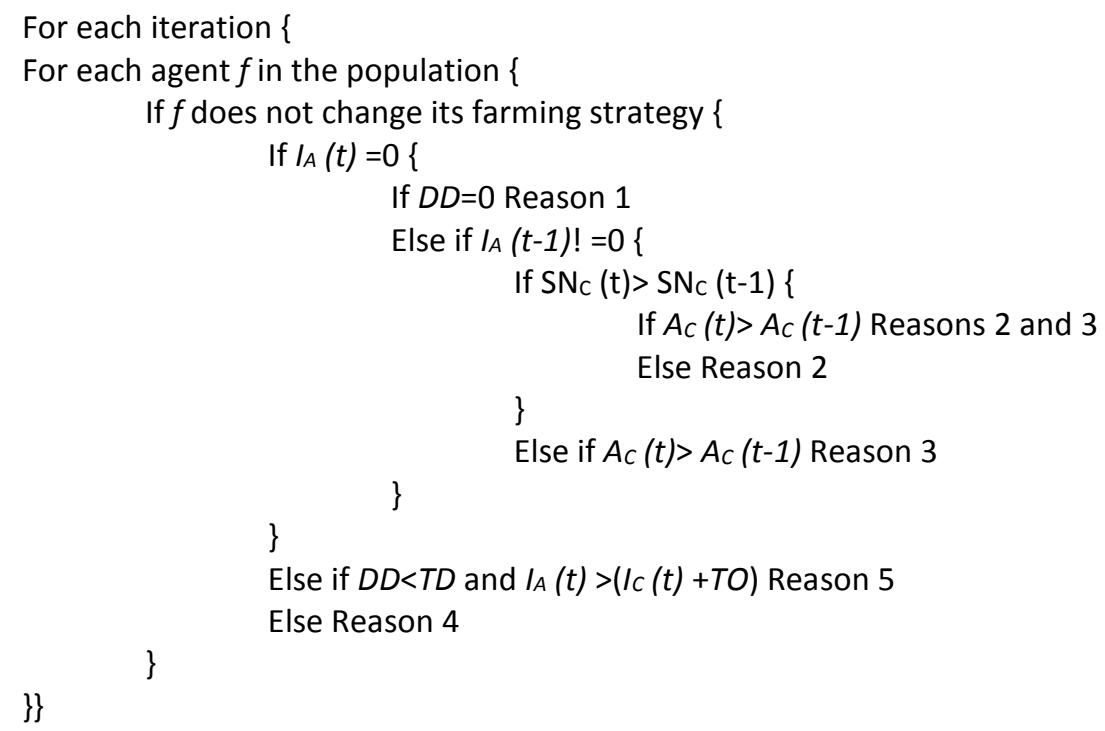

Algorithm 2-Overview of how reasons are identified. $I_{A}(t)$ is satisfaction with the alternative farming strategy at time $t, I_{A}(t-1)$ is satisfaction with the alternative farming strategy at time $t-1, I_{C}(t)$ is satisfaction with the current farming strategy at time $t$, DD is an agent's dissatisfaction duration, $T D$ is the threshold of dissatisfaction duration for changing farming strategy, $S N_{C}(t)$ is the subjective norm with the current farming strategy at time $t, A_{C}(t)$ is the attitude on the current farming strategy at time $t, T O$ is threshold of $I_{A}$ - $I_{C}$ to change farming strategy. 